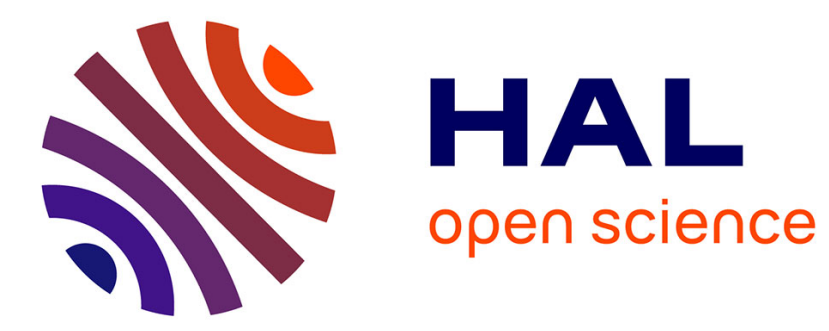

\title{
Deformation analysis of functionally graded beams by the direct approach
}

Mircea Bîrsan, Holm Altenbach, Tomasz Sadowski, Victor A. Eremeyev, Daniel Pietras

\section{- To cite this version:}

Mircea Bîrsan, Holm Altenbach, Tomasz Sadowski, Victor A. Eremeyev, Daniel Pietras. Deformation analysis of functionally graded beams by the direct approach. Composites Part B: Engineering, 2012, 43 (3), pp.1315-1328. hal-00826372

\section{HAL Id: hal-00826372}

\section{https://hal.science/hal-00826372}

Submitted on 28 May 2013

HAL is a multi-disciplinary open access archive for the deposit and dissemination of scientific research documents, whether they are published or not. The documents may come from teaching and research institutions in France or abroad, or from public or private research centers.
L'archive ouverte pluridisciplinaire HAL, est destinée au dépôt et à la diffusion de documents scientifiques de niveau recherche, publiés ou non, émanant des établissements d'enseignement et de recherche français ou étrangers, des laboratoires publics ou privés. 


\title{
Deformation analysis of functionally graded beams by the direct approach
}

\author{
M. Bîrsan ${ }^{\mathrm{a}, \mathrm{c}, *}, \mathrm{H}$. Altenbach $^{\mathrm{b}}, \mathrm{T}$. Sadowski ${ }^{\mathrm{c}}$, V.A. Eremeyev ${ }^{\mathrm{b}, \mathrm{d}}$, D. Pietras ${ }^{\mathrm{c}}$ \\ a Department of Mathematics, University "A.L Cuza" of Iași, 700506 Iași, Romania \\ Institut für Mechanik, Otto-von-Guericke-Universität Magdeburg. Universitätsplatz 2, 39106 Magdeburg, Germany \\ ${ }^{c}$ Facuity of Civil Engineering and Architecture, Lublin University of Technology, 20-618 Lublin, Poland \\ South Scientific Center of RASci Gr South Federal University, Rostov on Don, Russia
}

\begin{abstract}
A B S T R A C T
In this paper we employ the direct approach to the theory of rods and beams, which is based on the deformable curve model with a triad of rotating directors attached to each point. We show that this model (also called directed curve) is an efficient approach for analyzing the deformation of elastic beams with a complex material structure. Thus, we consider non homogeneous, composite and functionally graded beams made of isotropic or orthotropic materials and we determine the effective stiffness prop erties in terms of the three dimensional elasticity constants. We present general analytical expressions of the effective stiffness coefficients, valid for beams of arbitrary cross section shape. Finally, we apply this method for FGM beams made of metal foams and compare our analytical results with the numerica results obtained by a finite element analysis.
\end{abstract}

\section{Introduction}

Nowadays, the rod and beam structures made of functionally graded or non homogeneous materials are widely used in engi neering applications. One of the convenient ways to describe the mechanical properties of composite rods is to use the direct ap proach, which is based on the deformable curve model.

The classical way to derive theories of beams and rods is to use the thinness hypothesis and to perform an approximate analysis of the stress strain state of three dimensional bodies for which two dimensions are much smaller in comparison with the third one Thus, the derivation of a set of one dimensional approximate equa tions can be achieved by the application of kinematical or stress hypotheses, and the use of mathematical techniques like series expansions and asymptotic analysis [ 15$]$. Theory, finite element analysis, and various applications of FGM rods and beams are pre sented in many works, see e.g. [6 612$]$.

As an alternative to these methods, one can follow the direct ap proach by considering a deformable curve endowed with a certain microstructure, as a model for rods. The direct approach, which is well known since Euler, was summarized for the first time in the monograph of the Cosserat brothers [13] presenting the kinematical

\footnotetext{
* Corresponding author at: Department of Mathematics, University "A.I. Cuza" of Iaşi, 700506 Iaşi, Romıania. Tel.: +40 232201226; fax: +40232201160.

E-mail addresses: bmircea@uac.ro (M. Birsan), holmaltenbach@ovgu.de $(\mathrm{H}$ Altenbach), t.sadowski@pollub.pl (T. Sadowski), eremeyev.victor@gmail.com (V.A. Eremeyev).
}

model of a continuum with material points which behave like rigid bodies (having 6 degrees of freedom, instead of 3 in the classical con tinuum mechanics). Later, this idea has been developed by Ericksen and Truesdell [1.4], Green and Naghdi $[15,16]$ within the so called theory of Cosserat curves $[17,18]$. A different direct approach for shells and rods has been presented by Zhilin [19 21], who elaborated the so called theory of directed curves and surfaces. This theory follows the original idea of Cosserat and considers deform able continua (surfaces or curves) endowed with a triad of rigidly rotating orthonormal vectors connected to each point. This kinemat ical model was supplemented with appropriate constitutive equa tions [ 2022$]$, thus making the theory applicable to solve practical problems for rods.

In this paper we show that the model of directed curves is an efficient approach for analyzing elastic beams and rods with a complex internal structure (functionally graded, composite, non homogeneous, etc.). In order to be able to describe the complex mechanical behavior of functionally graded or composite beams and rods, we need to employ a quite general set of constitutive equations, which allows for the coupling of the extensional shear and bending torsion deformations. The structure of the constitu tive tensors and the form of the constitutive equations are pre sented in Section 2.

The main difficulty in any direct approach is the determination of the effective stiffness coefficients appearing in the one dimen sional constitutive equations in terms of the three dimensional elasticity constants. The determination of effective stiffness coeffi cients is important because it allows to reduce the treatment of 
three dimensional problems to much simpler one dimensional problems. To identify these mechanical properties for general non homogeneous rods, we compare the solutions of extension, bending and torsion problems in the direct approach with the cor responding results from the three dimensional theory $[23,24]$. Thus, we obtain the effective bending stiffness, extensional stiff ness, torsional rigidity and other coupling coefficients. Also, to determine the effective shear stiffness, we compare the shear vibrations of rectangular beams in the two approaches (direct and three dimensional). These results are presented in Sections 3 and 4 in the case of isotropic non homogeneous beams with arbi trary cross section shape. In Sections 5 and 6 we consider beams composed of two different non homogeneous materials, either orthotropic or isotropic, and we derive general formulas for the effective stiffness coefficients. These formulas are expressed in terms of the solutions to some auxiliary plane strain boundary va lue problems defined on the cross section domain. In general, the solutions of these auxiliary boundary value problems are not easy to find in a closed form, but we present in Section 7 some special cases for the geometry/material parameters in which we can ob tain the results in closed form. In Section 8 we employ our analyt ical modeling to analyze the deformation of FGM beams made of metal foams. The mass density distribution of the cellular material in the beam is given by a power law function of the cross section coordinate, while the Young's modulus is expressed by the Gib son Ashby formula for closed cell aluminum foams [25]. Finally, we verify our analytical modeling by comparing the results ob tained in the direct approach of FGM beams with the numerical solution of various bending problems obtained by a finite element analysis using ABAQUS.

The close agreement between the analytical and numerical solutions indicates that the direct approach to rods, together with the formulas for the effective stiffness coefficients derived in this paper, represent an efficient tool for the analysis of the deforma tion of functionally graded rods.

\section{Equations for curved rods in the direct approach}

\subsection{Material independent equations}

In this expository section we present the basic non linear equa tions for beams and rods, obtained by the direct approach in $[20,21]$. In this approach, the thin body is modeled as a deformable curve endowed with a triad of rigidly rotating vectors attached to each point.

We denote by $\mathcal{C}_{0}$ the deformable curve in its reference (ini tial) configuration and by $s$ the material coordinate along $\mathcal{C}_{0}$, which is also the arclength parameter. The position of the direc ted curve is described by the position vector $\boldsymbol{r}(s)$ and the at tached vectors $\boldsymbol{d}_{i}(s), i=1,2,3$, also called directors. The unit vectors $\boldsymbol{d}_{i}(s)$ are mutually orthogonal and they are chosen such that $\boldsymbol{d}_{3}$ coincides with the unit tangent $\boldsymbol{t} \equiv \boldsymbol{r}^{\prime}$, and $\boldsymbol{d}_{1}, \boldsymbol{d}_{2}$ belong to the normal plane to the curve $\mathcal{C}_{0}$. The rotations of the at tached triad of directors describe the rotations of the rod's cross sections during deformation.

Let $\mathcal{C}$ be the deformed configuration of the rod at time $t$, which is characterized by the vector fields (see Fig. 1)

\section{$\begin{array}{llll}\boldsymbol{R} & \boldsymbol{R}(s, t), \quad \boldsymbol{D}_{i} \quad \boldsymbol{D}_{i}(s, t), \quad i \quad 1,2,3,\end{array}$}

where $\boldsymbol{R}$ is the position vector and $\boldsymbol{D}_{i}$ are the directors after defor mation. We have $\boldsymbol{D}_{i} \quad \boldsymbol{D}_{j}=\delta_{i j}$ (the Kronecker symbol), but $\boldsymbol{D}_{3}$ is not tangent to the curve $\mathcal{C}$, i.e. the initial cross sections are no longer normal to the middle curve after deformation. In this model it is as sumed that the cross sections of the beam do not deform, but they only rotate with respect to the middle curve.

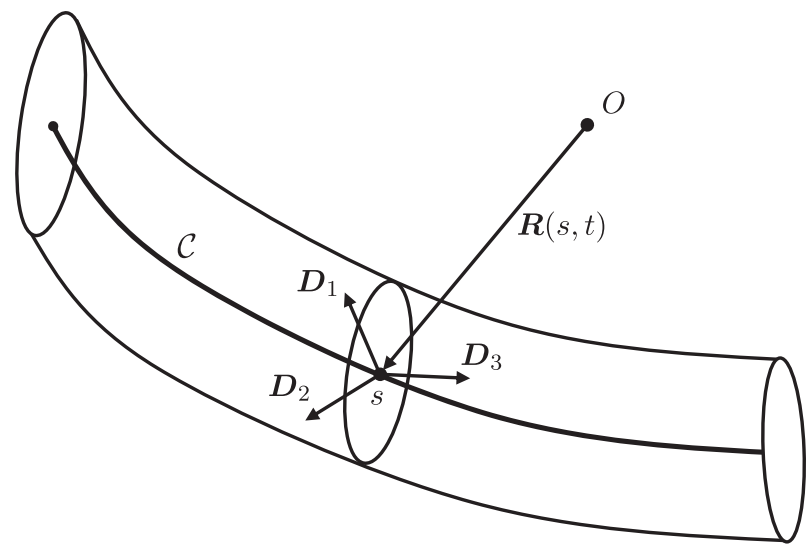

Fig. 1. The deformed configuration of the rod.

Let $\boldsymbol{P}(s, t)=\boldsymbol{D}_{k}(s, t) \otimes \boldsymbol{d}_{k}(s)$ be the rotation tensor. We employ throughout the Einstein's summation convention and the direct tensor notation in the sense of $[26,27]$. Greek indices range over the set $\{1,2\}$, while Latin indices take the values $\{1,2,3\}$. Denote by a superposed dot the material time derivative and by ()$^{\prime} \frac{\mathrm{d}}{\mathrm{ds}}$.

The velocity vector is $\boldsymbol{V}(s, t) \quad \dot{\boldsymbol{R}}(s, t)$, and the angular velocity vector $\boldsymbol{\omega}(s, t)$ is determined by the relation $\dot{\boldsymbol{P}} \boldsymbol{\omega} \times \boldsymbol{P}$, i.e. $\boldsymbol{\omega}$ is the axial vector of the antisymmetric tensor $\dot{\boldsymbol{P}} \boldsymbol{P}^{T}$. The equations of motion for rods are

$$
\begin{aligned}
& \boldsymbol{N}^{\prime}(s, t)+\rho_{0} \mathcal{F} \quad \rho_{0}\left(\boldsymbol{V}+\boldsymbol{\Theta}_{1} \cdot \boldsymbol{\omega}\right)^{\bullet}, \\
& \boldsymbol{M}^{\prime}(s, t)+\boldsymbol{R}^{\prime} \times \boldsymbol{N}(s, t)+\rho_{0} \mathcal{L} \quad \rho_{0}\left[\boldsymbol{V} \times \boldsymbol{\Theta}_{1} \cdot \boldsymbol{\omega}+\left(\boldsymbol{V} \cdot \boldsymbol{\Theta}_{1}+\boldsymbol{\Theta}_{2} \cdot \boldsymbol{\omega}\right)^{\bullet}\right],
\end{aligned}
$$

where $\boldsymbol{N}$ is the force vector, $\boldsymbol{M}$ is the moment vector, $\mathcal{F}$ and $\mathcal{L}$ are the external body force and moment per unit mass, $\rho_{0}$ is the mass density per unit length of $\mathcal{C}_{0}$, while the second order tensors $\boldsymbol{\Theta}_{1}(s, t)$ and $\boldsymbol{\Theta}_{2}(s, t)$ are the inertia tensors per unit mass. According to [21], the tensors $\boldsymbol{\Theta}_{\alpha}$ are expressed by $\boldsymbol{\Theta}_{\alpha}(s, t) \quad \boldsymbol{P}(s, t) \boldsymbol{\Theta}_{\alpha}^{0}(s)$ $\boldsymbol{P}^{\mathrm{T}}(s, t)$, where $\boldsymbol{\Theta}_{\alpha}^{0}(s)$ are the inertia tensors in the reference config uration, which are given by

$$
\begin{aligned}
& \rho_{0} \Theta_{1}^{0} \quad \int_{\Sigma}(\mathbf{1} \times \boldsymbol{a}) \rho^{*} \tilde{\mu} \mathrm{d} x_{1} \mathrm{~d} x_{2}, \\
& \rho_{0} \Theta_{2}^{0} \quad \int_{\Sigma}[(\boldsymbol{a} \cdot \boldsymbol{a}) \mathbf{1} \quad \boldsymbol{a} \otimes \boldsymbol{a}] \rho^{*} \tilde{\mu} \mathrm{d} x_{1} \mathrm{~d} x_{2} .
\end{aligned}
$$

Here $\rho^{*}$ is the mass density in the three dimensional rod, $\mathbf{1}$ is the second order unit tensor, $\Sigma$ is the domain of the cross section in the normal plane, $\boldsymbol{a}=x_{1} \boldsymbol{d}_{1}+x_{2} \boldsymbol{d}_{2}$ and $\mu \equiv 1+\frac{\boldsymbol{a} \cdot \boldsymbol{n}}{R_{c}}$, where $R_{c}$ is the ra dius of curvature of the curve $\mathcal{C}_{0}$ and $\boldsymbol{n}$ is the principal normal unit vector. In the case of straight rods, we have clearly $\mu 1$. In the general case of curved rods, since the diameter of the rod is much smaller than $R_{c}$, we have $\frac{|\boldsymbol{a} \cdot \boldsymbol{n}|}{R_{c}} \ll 1$, and thus $\mu>0$ and $\mu$ has a value close to 1 .

We note that $\boldsymbol{\Theta}_{1}$ is antisymmetric, $\boldsymbol{\Theta}_{2}$ is symmetric. The fields $\mathcal{F}$ and $\mathcal{L}$ account also for the loads acting on the lateral surface of three dimensional rods.

The vectors of deformation are defined as follows: the vector of extension shear $\mathcal{E} \quad \boldsymbol{R}^{\prime} \quad \boldsymbol{P} \boldsymbol{t}$, and the vector of bending tor sion $\boldsymbol{\Phi}$ is given by $\boldsymbol{P}^{\prime}=\boldsymbol{\Phi} \times \boldsymbol{P}$, i.e. $\boldsymbol{\Phi}$ is the axial vector of the antisymmetric tensor $\boldsymbol{P}^{\prime} \boldsymbol{P}^{\mathrm{T}}$. We also introduce the energetic vectors of deformation $\mathcal{E}_{*}$ and $\boldsymbol{\Phi}_{*}$ defined by $\mathcal{E}_{*} \quad \boldsymbol{P}^{T} \mathcal{E}$ and $\boldsymbol{\Phi}_{*}=\boldsymbol{P}^{T} \boldsymbol{\Phi}[20,21]$.

For general elastic beams, the constitutive assumptions imply that the internal energy density $\mathcal{U}$ is a function of the following arguments $\left\{\mathcal{E}_{*}, \boldsymbol{\Phi}_{*}\right\}$. In our work we consider that the internal en ergy is a quadratic function of its arguments. Thus, we have the fol lowing constitutive equations 
$\rho_{0} \mathcal{U} \quad \mathcal{U}_{0}+\boldsymbol{N}_{0} \cdot \mathcal{E}_{*}+\boldsymbol{M}_{0} \cdot \boldsymbol{\Phi}_{*}+\frac{1}{2} \mathcal{E}_{*} \cdot \boldsymbol{A} \cdot \mathcal{E}_{*}+\mathcal{E}_{*} \cdot \boldsymbol{B} \cdot \boldsymbol{\Phi}_{*}+\frac{1}{2} \boldsymbol{\Phi}_{*} \cdot \boldsymbol{C} \cdot \boldsymbol{\Phi}_{*}$, $\boldsymbol{N} \quad \frac{\partial\left(\rho_{0} \mathcal{U}\right)}{\partial \mathcal{E}_{*}} \cdot \boldsymbol{P}^{T}, \quad \boldsymbol{M} \quad \frac{\partial\left(\rho_{\mathcal{U}} \mathcal{U}\right)}{\partial \mathbf{\Phi}_{*}} \cdot \boldsymbol{P}^{T}$,

where $\mathcal{U}_{0}$ is a scalar, $\boldsymbol{N}_{0}, \boldsymbol{M}_{0}$ are vectors, and $\boldsymbol{A}, \boldsymbol{B}, \boldsymbol{C}$ are second order tensors, defined on the reference configuration. The structure and significance of the elasticity tensors $\boldsymbol{A}, \boldsymbol{B}$ and $\boldsymbol{C}$ have been discussed in $[20,21]$.

\subsection{Structure of constitutive tensors}

In our study we are interested to determine the structure of constitutive tensors for beams and rods made of functionally graded materials. We assume that the material properties do not vary along the length of the beam, but only across the cross sec tions. In other words, they depend on $\left(x_{1}, x_{2}\right)$, but not on $s$. In each cross section we chose the directors $\boldsymbol{d}_{1}$ and $\boldsymbol{d}_{2}$ along the principal axes of inertia. Thus, we have

$\left\langle\rho^{*} x_{1}\right\rangle \quad\left\langle\rho^{*} x_{2}\right\rangle \quad 0, \quad\left\langle\rho^{*} x_{1} x_{2}\right\rangle \quad 0$,

where we denote by $\langle f\rangle \quad \int_{\Sigma} f \mathrm{~d} x_{1} \mathrm{~d} x_{2}$ for any field $f$.

The structure of the constitutive tensors can be determined using the generalized theory of tensor symmetry $[21,28]$. In the general case of curved rods, the constitutive tensors depend on the geometry of the rod through the Darboux vector $\tau$ of the curve $\mathcal{C}_{0}$, and through the angle of natural twisting $\sigma=\varangle\left(\boldsymbol{d}_{1}, \boldsymbol{n}\right)$. The expressions of $\boldsymbol{A}, \boldsymbol{B}$ and $\boldsymbol{C}$ for homogeneous curved rods are pre sented in $[20,21]$. If we restrict for simplicity to straight rods with out natural twisting, then we have $\tau=\mathbf{0}$ and $\sigma=0$. Imposing that the orthogonal tensor $\mathbf{1} 2 \boldsymbol{t} \otimes \boldsymbol{t}$ belongs to the symmetry group of any constitutive tensor, we find that for non homogeneous rods $\boldsymbol{A}, \boldsymbol{B}$ and $\boldsymbol{C}$ have the following structures

$$
\begin{array}{ll}
\boldsymbol{A} & A_{1} \boldsymbol{d}_{1} \otimes \boldsymbol{d}_{1}+A_{2} \boldsymbol{d}_{2} \otimes \boldsymbol{d}_{2}+A_{3} \boldsymbol{t} \otimes \boldsymbol{t}+A_{12}\left(\boldsymbol{d}_{1} \otimes \boldsymbol{d}_{2}+\boldsymbol{d}_{2} \otimes \boldsymbol{d}_{1}\right), \\
\boldsymbol{B} & B_{13} \boldsymbol{d}_{1} \otimes \boldsymbol{t}+B_{31} \boldsymbol{t} \otimes \boldsymbol{d}_{1}+B_{23} \boldsymbol{d}_{2} \otimes \boldsymbol{t}+B_{32} \boldsymbol{t} \otimes \boldsymbol{d}_{2}, \\
\boldsymbol{C} & C_{1} \boldsymbol{d}_{1} \otimes \boldsymbol{d}_{1}+C_{2} \boldsymbol{d}_{2} \otimes \boldsymbol{d}_{2}+C_{3} \boldsymbol{t} \otimes \boldsymbol{t}+C_{12}\left(\boldsymbol{d}_{1} \otimes \boldsymbol{d}_{2}+\boldsymbol{d}_{2} \otimes \boldsymbol{d}_{1}\right) .
\end{array}
$$

Remark. The structure of the constitutive tensors can be derived also in the more general case of rods with natural twisting. In this case, the constitutive coefficients depend also on the angle of natural twist $\sigma(s)$, and the expressions corresponding to (6) have to be supplemented with additional terms.

Our aim is to determine the constitutive coefficients $A_{i}, C_{i}, A_{12}$, $C_{12}, B_{\alpha 3}$ and $B_{3 \alpha}$ for functionally graded beams and rods, in terms of the three dimensional elastic properties. These coefficients de scribe the effective stiffness properties of thin beams and rods. Since the constitutive coefficients do not depend on the deforma tion, their expressions can be derived by comparison of exact solu tions for directed curves with the results from three dimensional elasticity in the framework of linear theory.

In order to realize such comparison of exact solutions, we re strict ourselves to the linear theory. Let us note that in the the ory of beams and rods there is long tradition of using linear elasticity to derive one dimensional beams and rods theories including some non linear effects. This tradition is based on the fact that one can calculate stiffness parameters of beam or rod on the base of linear elasticity and then use the stiffness moduli in geometrically non linear theory of beams and rods. In deed, the coefficients of the strain energy density considered as the quadratic function of strain measures coincide for linear and for geometrically non linear theories of beams and rods. This fact is used for example in $[3,20,21]$ where different approaches are applied. In the paper the geometrically non linear approach with physically linear constitutive relations is considered. Such a theory can be applied for standard material. Exception is, for example, a rubber like material for which the quadratic form of the strain energy density is not valid in the case of large deformations, in general. Some recent attempts to apply non lin ear elasticity to construction of one dimensional theories of beams and rods are given for example in [29 37].

\section{Linearized equations for directed curves}

\subsection{Geometrical linearization}

In the linear setting, the displacement $\boldsymbol{u}(s, t)=\boldsymbol{R}(s, t) \quad \boldsymbol{r}(s)$ is as sumed to be infinitesimal. Also, the rotation tensor can be repre sented as $\boldsymbol{P}=\mathbf{1}+\boldsymbol{\psi} \times \mathbf{1}$, where $\boldsymbol{\psi}(s, t)$ is the vector of small rotations. The field $\psi$, which is assumed to be infinitesimal, satis fies the relations $\dot{\psi} \quad \boldsymbol{\omega}$ and $\psi^{\prime}=\boldsymbol{\Phi}$. The vectors of deformation are denoted in the linear case by $\boldsymbol{e}$ and $\boldsymbol{\kappa}$, and they are given by

$\boldsymbol{e} \equiv \boldsymbol{u}^{\prime}+\boldsymbol{t} \times \boldsymbol{\psi} \quad \mathcal{E} \quad \mathcal{E}_{*}, \quad \boldsymbol{\kappa} \equiv \boldsymbol{\psi}^{\prime} \quad \boldsymbol{\Phi} \quad \boldsymbol{\Phi}_{*}$.

The constitutive Eq. (4) reduce to

$$
\begin{aligned}
& \rho_{0} \mathcal{U}(\boldsymbol{e}, \boldsymbol{\kappa}) \quad \frac{1}{2} \boldsymbol{e} \cdot \boldsymbol{A} \cdot \boldsymbol{e}+\boldsymbol{e} \cdot \boldsymbol{B} \cdot \boldsymbol{\kappa}+\frac{1}{2} \boldsymbol{\kappa} \cdot \boldsymbol{C} \cdot \boldsymbol{\kappa}, \\
& \boldsymbol{N} \quad \frac{\partial\left(\rho_{0} \mathcal{U}\right)}{\partial \boldsymbol{e}}, \quad \boldsymbol{M} \quad \frac{\partial\left(\rho_{0} \mathcal{U}\right)}{\partial \boldsymbol{\kappa}} .
\end{aligned}
$$

The equations of motion (2) simplify to the forms

$$
\begin{aligned}
\boldsymbol{N}^{\prime}+\rho_{0} \mathcal{F} \quad & \rho_{0}\left(\ddot{\boldsymbol{u}}+\boldsymbol{\Theta}_{1}^{0} \cdot \ddot{\boldsymbol{\psi}}\right), \quad \boldsymbol{M}^{\prime}+\boldsymbol{t} \times \boldsymbol{N}+\rho_{0} \mathcal{L} \\
& \rho_{0}\left(\ddot{\boldsymbol{u}} \cdot \boldsymbol{\Theta}_{1}^{0}+\boldsymbol{\Theta}_{2}^{0} \cdot \ddot{\psi}\right) .
\end{aligned}
$$

To the governing field Eqs. (7) (9) we adjoin boundary conditions and initial conditions. Let $l$ be the length of the rod, so that the arc length parameter range over the interval $s \in[0, l]$. We denote the two endpoints by $\bar{s}_{1} \quad 0$ and $\bar{s}_{2} \quad l$ for convenience, and we consider boundary conditions of the type

$$
\begin{array}{lllllll}
\boldsymbol{u}\left(s_{\gamma}, t\right) & \boldsymbol{u}^{(\gamma)}(t) & \text { or } & \boldsymbol{N}\left(s_{\gamma}, t\right) & \boldsymbol{N}^{(\gamma)}(t), & \text { for } \gamma & 1,2, \\
\boldsymbol{\psi}\left(s_{\gamma}, t\right) & \boldsymbol{\psi}^{(\gamma)}(t) & \text { or } & \boldsymbol{M}\left(s_{\gamma}, t\right) & \boldsymbol{M}^{(\gamma)}(t), & \text { for } \gamma & 1,2 .
\end{array}
$$

The initial conditions are

$\begin{array}{lllll}\boldsymbol{u}(s, 0) & \boldsymbol{u}_{0}(s), & \dot{\boldsymbol{u}}(s, 0) & \boldsymbol{v}_{0}(s), \quad \psi(s, 0) & \psi_{0}(s),\end{array}$

$\dot{\psi}(s, 0) \quad \omega_{0}(s)$,

where the functions $\boldsymbol{u}_{0}, \boldsymbol{\psi}_{0}, \boldsymbol{v}_{0}, \omega_{0}$, as well as $\boldsymbol{u}^{(\gamma)}, \boldsymbol{\psi}^{(\gamma)}, \boldsymbol{N}^{(\gamma)}, \boldsymbol{M}^{(\gamma)}$ are prescribed.

The correspondence between the displacement and rotation fields $\{\boldsymbol{u}, \boldsymbol{\psi}\}$ for directed curves and the displacement vector $\boldsymbol{u}^{*}$ for three dimensional rods is established by the following relations [21]

$$
\begin{aligned}
\rho_{0}\left(\boldsymbol{u}+\Theta_{1}^{0} \cdot \psi\right) & \left\langle\rho^{*} \boldsymbol{u}^{*} \tilde{\mu}\right\rangle, \quad \rho_{0}\left(\boldsymbol{u} \cdot \Theta_{1}^{0}+\Theta_{2}^{0} \cdot \psi\right) \\
& \left\langle\rho^{*}\left(\boldsymbol{a} \times \boldsymbol{u}^{*}\right) \tilde{\mu}\right\rangle .
\end{aligned}
$$

Also, the relations between the fields $\{\boldsymbol{N}, \boldsymbol{M}\}$ and the Cauchy stress tensor $\boldsymbol{T}^{*}$ from three dimensional theory are given by

$$
\boldsymbol{N} \quad\left\langle\boldsymbol{t} \cdot \boldsymbol{T}^{*}\right\rangle, \quad \boldsymbol{M} \quad\left\langle\boldsymbol{a} \times\left(\boldsymbol{t} \cdot \boldsymbol{T}^{*}\right)\right\rangle .
$$

These relations are useful when comparing the solutions of some problems in the two different approaches.

\subsection{Straight rods}

In what follows we restrict our attention to straight rods without natural twisting. In this case, we can chose the Cartesian 
coordinate frame $O x_{1} x_{2} x_{3}$ such that the curve $\mathcal{C}_{0}$ is situated on the axis $O x_{3}$, between the limits $x_{3}=0, l$, and we have

$\begin{array}{llllllllllllll}\boldsymbol{t} & \boldsymbol{d}_{3} & \boldsymbol{e}_{3}, & \boldsymbol{n} & \boldsymbol{d}_{1} & \boldsymbol{e}_{1}, & \boldsymbol{d}_{2} & \boldsymbol{e}_{2}, & s & x_{3}, & \mu & 1, & \boldsymbol{\Theta}_{1}^{0} & \mathbf{0},\end{array}$ $\rho_{0} \Theta_{2}^{0} \quad I_{1} \boldsymbol{e}_{1} \otimes \boldsymbol{e}_{1}+I_{2} \boldsymbol{e}_{2} \otimes \boldsymbol{e}_{2}+\left(I_{1}+I_{2}\right) \boldsymbol{e}_{3} \otimes \boldsymbol{e}_{3}, I_{1} \quad\left\langle\rho^{*} x_{2}^{2}\right\rangle, I_{2} \quad\left\langle\rho^{*} x_{1}^{2}\right\rangle$,

where $\boldsymbol{e}_{i}$ denote the unit vectors along $O x_{i}$.

To distinguish between the extensional, torsional, bending, and shear deformation, we decompose the vectors $\boldsymbol{u}, \boldsymbol{\psi}, \boldsymbol{e}, \boldsymbol{\kappa}, \boldsymbol{N}$, $\boldsymbol{M}, \mathcal{F}$ and $\mathcal{L}$ by the tangent direction $\boldsymbol{t}$ and the normal plane $\left(\boldsymbol{e}_{1}\right.$, $\left.\boldsymbol{e}_{2}\right):$

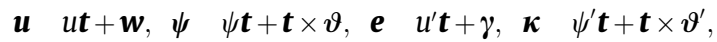

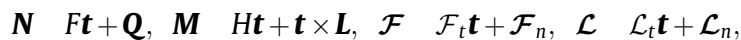

with $\boldsymbol{\gamma}=\boldsymbol{w}^{\prime} \quad \boldsymbol{\vartheta}$. The vectors $\boldsymbol{w}, \boldsymbol{\vartheta}, \boldsymbol{\gamma}, \boldsymbol{Q}, \boldsymbol{L}, \mathcal{F}_{n}$ and $\mathcal{L}_{n}$ are orthog onal to $\boldsymbol{t}$. Here $\boldsymbol{\gamma}$ is the transverse shear vector, $u$ is the longitudinal displacement, $\boldsymbol{w}=w_{\alpha} \boldsymbol{e}_{\alpha}$ is the vector of transversal displacement, $\psi$ is the torsion, $\boldsymbol{\vartheta}^{\prime} \quad \vartheta_{\alpha}^{\prime} \boldsymbol{e}_{\alpha}$ is the vector of bending deformation, $F$ is the longitudinal force, $\boldsymbol{Q}=Q_{\alpha} \boldsymbol{e}_{\alpha}$ is the vector of transversal force, $H$ is the torsion moment and $\boldsymbol{L}=L_{\alpha} \boldsymbol{e}_{\alpha}$ is the vector of bending mo ment. Using the decompositions (12) and the structure of constitu tive tensors (6), we remark that the constitutive Eq. (8) can be written in component form as

$Q_{1} \quad A_{1}\left(\begin{array}{ll}w_{1}^{\prime} & \vartheta_{1}\end{array}\right)+A_{12}\left(\begin{array}{ll}w_{2}^{\prime} & \vartheta_{2}\end{array}\right)+B_{13} \psi^{\prime}$,

$Q_{2} \quad A_{12}\left(\begin{array}{ll}w_{1}^{\prime} & \vartheta_{1}\end{array}\right)+A_{2}\left(\begin{array}{ll}w_{2}^{\prime} & \vartheta_{2}\end{array}\right)+B_{23} \psi^{\prime}$,

F $\quad A_{3} u^{\prime} \quad B_{31} \vartheta_{2}^{\prime}+B_{32} \vartheta_{1}^{\prime}, H \quad C_{3} \psi^{\prime}+B_{13}\left(w_{1}^{\prime} \vartheta_{1}\right)+B_{23}\left(\begin{array}{ll}w_{2}^{\prime} & \vartheta_{2}\end{array}\right)$,

$L_{1} \quad C_{2} \vartheta_{1}^{\prime} \quad C_{12} \vartheta_{2}^{\prime}+B_{32} u^{\prime}, L_{2} \quad C_{12} \vartheta_{1}^{\prime}+C_{1} \vartheta_{2}^{\prime} \quad B_{31} u^{\prime}$.

The constitutive coefficients are constants, since we consider rods made of non homogeneous materials which properties do not de pend on the axial coordinate $s$.

We observe that the general boundary initial value problem for non homogeneous rods does not decouple into sub problems. Note that in the case of homogeneous materials the general prob lem decouples into the extension torsion problem and the bend ing shear problem, see [21]. The relations of identification (10) and (11), written for straight rods, become

$$
\begin{array}{llllllll}
\rho_{0} w_{\alpha} & \left\langle\rho^{*} u_{\alpha}^{*}\right\rangle, & \rho_{0} u & \left\langle\rho^{*} u_{3}^{*}\right\rangle, & \rho_{0} & \left\langle\rho^{*}\right\rangle, \\
\vartheta_{1} & \frac{\left\langle\rho^{*} x_{1} u_{3}^{*}\right\rangle}{I_{2}}, & \vartheta_{2} & \frac{\left\langle\rho^{*} x_{2} u_{3}^{*}\right\rangle}{I_{1}}, & \psi & \left.\left.\frac{\left\langle\rho ^ { * } \left( x_{1} u_{2}^{*}\right.\right.}{I_{1}+I_{2}} x_{1} u_{1}^{*}\right)\right\rangle \\
Q_{\alpha} & \left\langle t_{3 \alpha}^{*}\right\rangle, \quad F & \left\langle t_{33}^{*}\right\rangle, & L_{\alpha} & \left\langle x_{\alpha} t_{33}^{*}\right\rangle, & H & \left\langle x_{1} t_{32}^{*}\right. & \left.x_{2} t_{31}^{*}\right\rangle,
\end{array}
$$

where $u_{i}^{*}$ and $t_{i j}^{*}$ are the components of $\boldsymbol{u}^{*}$ and $\boldsymbol{T}^{*}$, respectively. The relations (14) will be used to identify the corresponding fields in the two approaches (directed curves and three dimensional).

\subsection{Extension, bending and torsion in the direct approach}

Let us find the exact solution of the problem of extension, bend ing and torsion of directed curves. We mention that this solution is exact up to rigid body displacement and rotation fields. In the lin ear theory the rigid body fields have the general form $\boldsymbol{u} \quad \boldsymbol{a}+\boldsymbol{b} \times \boldsymbol{r}, \psi \quad \boldsymbol{b}$, where $\boldsymbol{a}$ and $\boldsymbol{b}$ are arbitrary constant vectors.

Let us determine the equilibrium of a straight rod subjected to an axial force $\bar{F}$, a torsion moment $\bar{H}$, and bending moments $\bar{L}_{\alpha}$ ap plied to both ends. The body forces and moments are absent. In our case, the equilibrium equations corresponding to (9) are

$Q_{\alpha}^{\prime}(s) \quad 0, \quad F^{\prime}(s) \quad 0, \quad L_{\alpha}^{\prime}(s)+Q_{\alpha}(s) \quad 0, \quad H^{\prime}(s) \quad 0$,

$s \in(0, l)$,

while the boundary conditions on the ends of the rods are
$Q_{\alpha}(0) \quad Q_{\alpha}(l) \quad 0, \quad F(0) \quad F(l) \quad \bar{F}$,
$L_{\alpha}(0) \quad L_{\alpha}(l) \quad \bar{L}_{\alpha}, \quad H(0) \quad H(l) \quad \bar{H}$.

Using the constitutive Eq. (13) we obtain a system of ordinary dif ferential equations which yields the solution

$w_{\alpha}(s) \quad \frac{1}{2} a_{\alpha} s^{2}+b_{\alpha} s, \quad u(s) \quad a_{3} s, \quad \vartheta_{\alpha}(s) \quad a_{\alpha} s$,
$\psi(s) \quad b_{3} s$,

where the constants $a_{i}$ and $b_{i}$ are determined by the algebraic linear systems

$\left[\begin{array}{ccc}C_{2} & C_{12} & B_{32} \\ C_{12} & C_{1} & B_{31} \\ B_{32} & B_{31} & A_{3}\end{array}\right]\left[\begin{array}{l}a_{1} \\ a_{2} \\ a_{3}\end{array}\right] \quad\left[\begin{array}{c}\bar{L}_{1} \\ \bar{L}_{2} \\ \bar{F}\end{array}\right],\left[\begin{array}{lll}A_{1} & A_{12} & B_{13} \\ A_{12} & A_{2} & B_{23} \\ B_{13} & B_{23} & C_{3}\end{array}\right]\left[\begin{array}{l}b_{1} \\ b_{2} \\ b_{3}\end{array}\right] \quad\left[\begin{array}{l}0 \\ 0 \\ \bar{H}\end{array}\right]$

The force and moment vector fields corresponding to this solution are given by

$\boldsymbol{N} \quad \bar{F} \boldsymbol{e}_{3}, \quad \boldsymbol{M} \quad \bar{L}_{2} \boldsymbol{e}_{1}+\bar{L}_{1} \boldsymbol{e}_{2}+\bar{H} \boldsymbol{e}_{3}$.

This solution will be used later for comparison with three dimen sional solutions, in order to identify the effective stiffness coeffi cients for non homogeneous thin rods.

\section{Determination of constitutive coefficients for isotropic rods}

\subsection{Deformation of non homogeneous three dimensional rods}

Let us consider a three dimensional rod which occupies the do main $\mathcal{B} \quad\left\{\left(x_{1}, x_{2}, x_{3}\right) \mid\left(x_{1}, x_{2}\right) \in \Sigma, x_{3} \in[0, l]\right\}$. The cross section $\Sigma$ is arbitrary and the symmetry relations (5) are satisfied. The body $\mathcal{B}$ is made of an isotropic and non homogenous material such that the mass density $\rho^{*}$ and the Lamé moduli $\lambda, \mu$ are independent of the axial coordinate, i.e. we have

$\rho^{*} \quad \rho^{*}\left(x_{1}, x_{2}\right), \quad \lambda \quad \lambda\left(x_{1}, x_{2}\right), \quad \mu \quad \mu\left(x_{1}, x_{2}\right)$.

We consider the deformation of such cylinders under the action of terminal forces and moments.

We assume that the body $\mathcal{B}$ is in equilibrium, in the absence of external body loads and tractions on the lateral surfaces. On the two ends of the cylinder act a resultant axial force and a resultant moment. We consider the same problem as in Section 3.4, but for mulated in the three dimensional setting. In view of the relations $(14)_{7} \quad 10$ we take the boundary conditions

$$
\begin{array}{lllll}
\left\langle t_{3 \alpha}^{*}\right\rangle & 0, \quad\left\langle t_{33}^{*}\right\rangle & \bar{F}, \quad\left\langle x_{\alpha} t_{33}^{*}\right\rangle & \bar{L}_{\alpha}, \\
\left\langle x_{1} t_{32}^{*}\right. & \left.x_{2} t_{31}^{*}\right\rangle & \bar{H} \text { for } x_{3} & 0, l .
\end{array}
$$

The solution of this three dimensional problem for non homoge neous rods is presented in [23] Section 3.3 and Section 3.4, where it is expressed in terms of the solutions to some auxiliary plane strain problems. For the sake of completeness and for later reference we present these three dimensional results.

We denote by $u_{\alpha}^{(1)}, u_{\alpha}^{(2)}$ and $u_{\alpha}^{(3)}$ the solutions of the 3 plane strain problems $\mathcal{D}^{(1)}, \mathcal{D}^{(2)}$ and $\mathcal{D}^{(3)}$ respectively, defined on the do main $\Sigma$ by

$$
\begin{aligned}
& \mathcal{D}^{(\gamma)}: \quad t_{\beta \alpha, \beta}^{(\gamma)}+\left(\lambda x_{\gamma}\right)_{\alpha} \quad 0 \quad \text { in } \Sigma, \quad t_{\beta \alpha}^{(\gamma)} n_{\beta}^{*} \quad \lambda x_{\gamma} n_{\alpha}^{*} \quad \text { on } \partial \Sigma \\
& \mathcal{D}^{(3)}: \quad t_{\beta \alpha, \beta}^{(3)}+\lambda_{, \alpha} \quad 0 \quad \text { in } \Sigma, \quad t_{\beta \alpha}^{(3)} n_{\beta}^{*} \quad \lambda n_{\alpha}^{*} \quad \text { on } \partial \Sigma \text {, }
\end{aligned}
$$

where obviously $t_{\alpha \beta}^{(k)} \quad \lambda u_{\rho, \rho}^{(k)} \delta_{\alpha \beta}+\mu\left(u_{\alpha, \beta}^{(k)}+u_{\beta, \alpha}^{(k)}\right),(k=1,2,3$ and $\alpha$, $\beta=1,2)$ and $\boldsymbol{n}^{*} n_{\alpha}^{*} \boldsymbol{e}_{\alpha}$ is the outward unit normal to $\partial \Sigma$. Let $\varphi\left(x_{1}, x_{2}\right)$ be the solution of the Neumann type boundary value problem

$\left(\mu \varphi_{, \alpha}\right)_{, \alpha} \quad \mu_{, 1} x_{2} \quad \mu_{, 2} x_{1} \quad$ in $\Sigma, \quad \frac{\partial \varphi}{\partial n^{*}} \quad x_{2} n_{1}^{*} \quad x_{1} n_{2}^{*} \quad$ on $\partial \Sigma$. 
The existence of solutions to the above boundary value problems (21) and (22) is proved in [23], Sections 3.2 and 3.4. Then, the solu tion of our three dimensional problem for the loads (20) is given by

$$
\begin{array}{ll}
u_{1} & \frac{1}{2} \hat{a}_{1} x_{3}^{2} \quad \tau x_{2} x_{3}+\sum_{k 1}^{3} \hat{a}_{k} u_{1}^{(k)}\left(x_{1}, x_{2}\right), \\
u_{2} & \frac{1}{2} \hat{a}_{2} x_{3}^{2}+\tau x_{1} x_{3}+\sum_{k 1}^{3} \hat{a}_{k} u_{2}^{(k)}\left(x_{1}, x_{2}\right), \\
u_{3} & \left(\hat{a}_{1} x_{1}+\hat{a}_{2} x_{2}+\hat{a}_{3}\right) x_{3}+\tau \varphi\left(x_{1}, x_{2}\right),
\end{array}
$$

where the constants $\tau$ and $\hat{a}_{i}$ are given by the relations

$\tau \quad \frac{\bar{H}}{D_{*}}$ and $D_{\alpha j} \hat{a}_{j} \quad \bar{L}_{\alpha}, \quad D_{3 j} \hat{a}_{j} \quad \bar{F}$.

Here the torsional rigidity $D_{*}$ is expressed by

$D_{*}\left\langle\mu\left[x_{1}\left(x_{1}+\varphi_{, 2}\right)+x_{2}\left(x_{2} \quad \varphi_{, 1}\right)\right]\right\rangle$,

while the coefficients $D_{i j}$ are given by

$$
\begin{array}{lllll}
D_{\alpha \beta} & \left\langle(\lambda+2 \mu) x_{\alpha} x_{\beta}+\lambda x_{\alpha} u_{\gamma, \gamma}^{(\beta)}\right\rangle, & D_{33} & \left\langle(\lambda+2 \mu)+\lambda u_{\gamma, \gamma}^{(3)}\right\rangle, \\
D_{\alpha 3} & \left\langle(\lambda+2 \mu) x_{\alpha}+\lambda x_{\alpha} u_{\gamma, \gamma}^{(3)}\right\rangle, & D_{3 \alpha} & \left\langle(\lambda+2 \mu) x_{\alpha}+\lambda u_{\gamma, \gamma}^{(\alpha)}\right\rangle .
\end{array}
$$

In [23], Section 3.3, it is shown that $D_{i j}=D_{j i}$ and $\operatorname{det}\left(D_{i j}\right)_{3 \times 3} \neq 0$, so that we can determine the constants $\hat{a}_{i}$ from the system $(24)_{2,3}$.

Remark. If we introduce the stress function $\chi\left(x_{1}, x_{2}\right)$ by the relations

$\chi_{, 1} \quad \mu\left(\varphi_{, 2}+x_{1}\right), \quad \chi_{, 2} \quad \mu\left(\varphi_{, 1} \quad x_{2}\right)$,

then the torsional rigidity is given by

$D_{*} \quad 2\left\langle\chi\left(x_{1}, x_{2}\right)\right\rangle$.

The stress function $\chi$ can be obtained from the boundary value problem

$$
\left(\frac{1}{\mu} \chi_{, \alpha}\right)_{, \alpha} \quad 2 \text { in } \Sigma, \quad \chi \quad 0 \text { on } \partial \Sigma
$$

provided that the domain $\Sigma$ is simply connected. In the case of mul tiply connected cross sections $\Sigma$, the torsion problem has been studied in, e.g., [38,39].

Let us compare now the three dimensional solution (23) with the solution (17) obtained in the direct approach to rods, taking into account the relations (5) and (14). By comparison, it follows that we have to identify the constants

$$
\begin{array}{llllllllll}
C_{3} & D_{*}, & A_{3} & D_{33}, & C_{1} & D_{22}, & C_{2} & D_{11}, & C_{12} & D_{12}, \\
B_{31} & D_{23}, & B_{32} & D_{13}, & B_{13} & B_{23} & 0 . & &
\end{array}
$$

Thus, from (26) (28) we obtain the following expressions for the constitutive coefficients

$$
\begin{array}{llll}
C_{3} & 2\left\langle\chi\left(x_{1}, x_{2}\right)\right\rangle, A_{3} & \left\langle(\lambda+2 \mu)+\lambda u_{\gamma, \gamma}^{(3)}\right\rangle, C_{1} & \left\langle(\lambda+2 \mu) x_{2}^{2}+\lambda x_{2} u_{\gamma, \gamma}^{(2)}\right\rangle, \\
C_{2} & \left\langle(\lambda+2 \mu) x_{1}^{2}+\lambda x_{1} u_{\gamma, \gamma}^{(1)}\right\rangle, C_{12} & \left\langle(\lambda+2 \mu) x_{1} x_{2}+\lambda x_{1} u_{\gamma, \gamma}^{(2)}\right\rangle, B_{\alpha 3} & 0, \\
B_{31} & \left\langle(\lambda+2 \mu) x_{2}+\lambda x_{2} u_{\gamma, \gamma}^{(3)}\right\rangle, B_{32} & \left\langle(\lambda+2 \mu) x_{1}+\lambda x_{1} u_{\gamma, \gamma}^{(3)}\right\rangle .
\end{array}
$$

By virtue of the identifications (14) and (29) we can verify that the fields $u, w_{\alpha}, \psi, \boldsymbol{N}$ and $\boldsymbol{M}$ calculated for the solutions in the two dif ferent approaches coincide.

Remark. For the fields $\vartheta_{\alpha}$ corresponding to the three dimensional solution (23) we obtain from (14) 1,2 and (5) the expressions

$$
\vartheta_{\alpha} \quad \hat{a}_{\alpha} x_{3} \quad \tau \frac{\left\langle\rho^{*} x_{\alpha} \varphi\right\rangle}{\left\langle\rho^{*} x_{\alpha}^{2}\right\rangle}, \quad \alpha \quad 1,2, \text { not summed. }
$$

Comparing this relation with the field $\vartheta_{\alpha}$ from the solution $(17)_{3}$ for directed curves, we see that we have to approximate $\frac{\left\langle\rho^{*} x_{\alpha} \varphi\right\rangle}{\left\langle\rho^{*} \chi_{\alpha}^{2}\right\rangle} \simeq 0, \quad \alpha \quad 1,2$, not summed

where $\varphi\left(x_{1}, x_{2}\right)$ is the torsion function given by (22). For example, in the case when $\Sigma$ is an elliptical domain $\Sigma \quad\left\{\left(x_{1}, x_{2}\right) \mid \frac{x_{1}^{2}}{a^{2}}+\frac{x_{2}^{2}}{b^{2}}<1\right\}$ and $\mu$ is constant, then we have $\varphi\left(x_{1}, x_{2}\right) \quad \frac{b^{2} a^{2}}{a^{2}+b^{2}} x_{1} x_{2}$ so that the above approximation is justified.

We remark that, due to the shear bending coupling in the case of static problems, the effective shear stiffness coefficients $A_{1}, A_{2}$ and $A_{12}$ cannot be obtained by analyzing static shear problems and using the same procedure as above. (For thin beams, the coef ficients $A_{1}, A_{2}, A_{12}$ will not enter in the leading order terms of the solutions.) For this reason, we determine the effective shear stiff ness coefficients by solving a free vibration problem. ${ }^{1}$ The neces sity of considering free vibration problems for the determination of effective shear stiffness properties is also discussed in details in [20] Section 6, and in [21, pp. 34 38].

\subsection{Shear vibrations of rectangular rods}

Consider a three dimensional rod which occupies the domain $\mathcal{R}\left\{\left(x_{1}, x_{2}, x_{3}\right) \mid x_{1} \in\left(\frac{a}{2}, \frac{a}{2}\right), x_{2} \in\left(\frac{b}{2}, \frac{b}{2}\right), x_{3} \in(0, l)\right\}$, made of a non homogeneous isotropic material. The material parameters $\lambda$, $\mu$ and $\rho^{*}$ are given functions of $\left(x_{1}, x_{2}\right)$. Assume that the mass den sity $\rho^{*}$ has a symmetrical distribution across the thickness: $\rho^{*}\left(x_{1}, x_{2}\right)=\rho^{*}\left(x_{1}, x_{2}\right)$.

The body loads are zero, the lateral surfaces $x_{1} \quad \pm \frac{a}{2}$ and $x_{2} \quad \pm \frac{b}{2}$ are traction free, and the end boundary conditions are given by

$\begin{array}{lllllllll}u_{1}^{*} & u_{2}^{*} & 0 & \text { and } & t_{33}^{*} & 0 & \text { for } & x_{3} & 0, l .\end{array}$

To determine the shear vibrations of this rod, we search for solu tions $\boldsymbol{u}^{*}$ of the form

$\boldsymbol{u}^{*} \quad W \cos (\omega t) \sin \left(\frac{\pi}{a} x_{1}\right) \boldsymbol{e}_{3}$,

where $W$ is a constant and $\omega$ is the lowest natural frequency. We observe that all the boundary conditions are satisfied by the field (31), and the equations of motion reduce to $t_{13,1}^{*} \quad \rho^{*} u_{3}^{*}$, which by integration with respect to $x_{1}$ gives

$t_{13}^{*} \quad W \omega^{2} \cos (\omega t) \int_{a / 2}^{x_{1}} \rho^{*}\left(x_{1}, x_{2}\right) \sin \left(\frac{\pi}{a} x_{1}\right) \mathrm{d} x_{1}$.

Using the constitutive equation for $t_{13}^{*}$ we get

$\mu\left(x_{1}, x_{2}\right) \frac{\pi}{a} \cos \left(\frac{\pi}{a} x_{1}\right) \quad \omega^{2} \int_{a / 2}^{x_{1}} \rho^{*}\left(x_{1}, x_{2}\right) \sin \left(\frac{\pi}{a} x_{1}\right) \mathrm{d} x_{1}$

We apply the mean value theorem for the integral in (32) and we deduce that there exists a point $\alpha \quad \alpha\left(x_{1}, x_{2}\right) \in\left(\frac{a}{2}, x_{1}\right)$ such that

$\int_{a / 2}^{x_{1}} \rho^{*}\left(x_{1}, x_{2}\right) \sin \left(\frac{\pi}{a} x_{1}\right) \mathrm{d} x_{1} \quad \rho^{*}\left(\alpha, x_{2}\right) \int_{a / 2}^{x_{1}} \sin \left(\frac{\pi}{a} x_{1}\right) \mathrm{d} x_{1}$.

Substituting (33) into (32) and integrating over $\Sigma$ we obtain

$\omega^{2} \quad\left(\frac{\pi}{a}\right)^{2} \frac{\left\langle\mu\left(x_{1}, x_{2}\right)\right\rangle}{\left\langle\rho^{*}\left(\alpha, x_{2}\right)\right\rangle}$.

Let us treat the same problem using the approach of directed curves. We consider a straight rod along the $O x_{3}$ axis for which the arclength parameter $s \in(0, l)$. The external body loads $\boldsymbol{F}$ and $\boldsymbol{L}$ are zero. According to (14) and (30) we have the following boundary conditions on the rod ends

\footnotetext{
${ }^{1}$ Note that the use of static and dynamic problems for identification purposes must result in the same effective stiffness properties. The type of the problem (static or dynamic) should not influence the final results [19].
} 
$w_{\alpha} \quad 0, \quad F \quad 0, \quad \psi \quad 0, \quad L_{\alpha} \quad 0 \quad(\alpha \quad 1,2), \quad$ for $s \quad 0, l . \quad(35)$

In order to study the shear vibrations, we search for solutions of the Eqs. (9), (13) of the form

$\vartheta_{1} \quad \bar{W} \cos (\omega t), \quad \vartheta_{2} \quad \psi \quad 0, \quad u \quad w_{\alpha} \quad 0$,

where $\bar{W}$ is a constant and $\bar{\omega}$ is the natural frequency of the rod. In view of the constitutive Eq. (13), we see that the boundary condi tions (35) are satisfied. Imposing that the fields (36) verify the equa tions of motion (9) we find

$\omega^{2} \quad \frac{A_{1}}{I_{2}}$ and $A_{12} \quad 0$.

We identify the natural frequencies $\omega$ and $\bar{\omega}$ from (34) and (36) ${ }_{1}$, and we obtain the expression of the constitutive coefficient $A_{1}$ as follows:

$A_{1} \quad k \frac{\langle\mu\rangle\left\langle\rho^{*} x_{1}^{2}\right\rangle \operatorname{Area}(\Sigma)}{\left\langle\rho^{*}\left(\alpha, x_{2}\right)\right\rangle\left\langle x_{1}^{2}\right\rangle} \quad$ with $k \frac{\pi^{2}}{12}$,

where the factor $k$ is similar to the shear correction factor intro duced first by Timoshenko [40] in the theory of beams (note that in the original contribution of Timoshenko the value is $2 / 3$ ). One can proceed analogously for the $x_{2}$ direction and find a similar expression for $A_{2}$. These relations express the transverse shear stiff ness coefficients for non homogeneous rectangular rods. The value given by (38) will be verified in Section 8, where we consider the bending of cantilever functionally graded beams and make a com parison with numerical results.

\section{Remarks.}

1. In the case of homogeneous rods, $\mu$ and $\rho^{*}$ are constant, and from (38) we get the well known formulas [20]

$$
A_{1} \quad A_{2} \quad k \mu \operatorname{Area}(\Sigma), \quad A_{12} \quad 0 .
$$

The value of the factor $k$ in relation (39) has been discussed in [21].

2 . In the case of thin rods, when $\rho^{*}$ has a smooth variation across the thickness, we can employ the approximation

$$
\left\langle\rho^{*}\left(\alpha, x_{2}\right)\right\rangle \simeq\left\langle\rho^{*}\left(x_{1}, x_{2}\right)\right\rangle .
$$

Then, we substitute (40) into (38) and find

$$
A_{\gamma} \quad k\langle\mu\rangle \frac{\left\langle\rho^{*} x_{\gamma}^{2}\right\rangle \operatorname{Area}(\Sigma)}{\left\langle\rho^{*}\right\rangle\left\langle x_{\gamma}^{2}\right\rangle} \quad(\gamma \quad 1,2 \text { not summed }), A_{12} \quad 0
$$

The simplified (approximate) formulas (41) can be used to estimate the transverse shear stiffness for arbitrary non homogeneous rods (not necessarily rectangular or symmetrical) in most cases.

\section{Beams composed of two different materials}

In this section we consider beams and rods made of two isotro pic and non homogeneous materials. The body $\mathcal{B}$ is decomposed in two regions $\mathcal{B}_{1}$ and $\mathcal{B}_{2}$ such that $\mathcal{B}_{\rho} \quad\left\{\left(x_{1}, x_{2}, x_{3}\right) \mid\left(x_{1}, x_{2}\right) \in S_{\rho}, x_{3}\right.$ $\in(0, l)\}$. Thus, the cross section $\Sigma$ is decomposed in two domains $S_{1}$ and $S_{2}$ with $S_{1} \cap S_{2}=\emptyset$, see Fig. 2a. We denote by $\Gamma_{0}$ the curve of separation between the domains $S_{1}$ and $S_{2}$ and by $\Gamma_{1}, \Gamma_{2}$ the complementary subsets of $\partial \Sigma$ such that $\partial S_{\rho}=\Gamma_{0} \cap \Gamma_{\rho}$. Let $\Pi_{0}=\left\{\left(x_{1}, x_{2}, x_{3}\right) \mid\left(x_{1}, x_{2}\right) \in \Gamma_{0}, x_{3} \in(0, l)\right\}$ be the surface of separation of the two materials. We assume that the two materials are welded together along $\Pi_{0}$ and there is no separation of material along $\Pi_{0}$, so we have the conditions

$\left[\boldsymbol{u}^{*}\right]_{1} \quad\left[\boldsymbol{u}^{*}\right]_{2}, \quad\left[\boldsymbol{T}^{*}\right]_{1} \cdot \boldsymbol{n}^{0} \quad\left[\boldsymbol{T}^{*}\right]_{2} \cdot \boldsymbol{n}^{0} \quad$ on $\Pi_{0}$,

where $\boldsymbol{n}^{0} \quad n_{\alpha}^{0} \boldsymbol{e}_{\alpha}$ is the unit normal of $\Pi_{0}$, outward to $\mathcal{B}_{1}$. The nota tions $[f]_{1}$ and $[f]_{2}$ represent the values of any field $f$ on $\Pi_{0}$, calculated

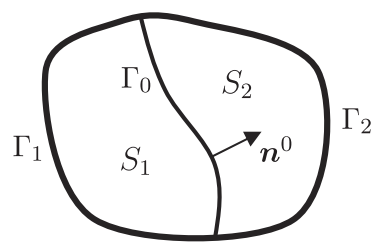

(a)

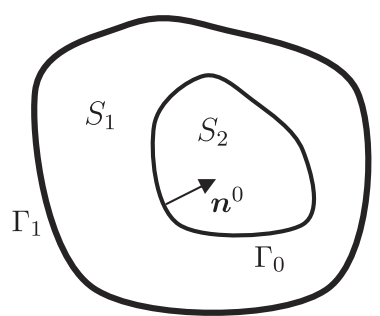

(b)
Fig. 2. The cross-section of rods composed of two materials.

as the limits of the values from the domains $\mathcal{B}_{1}$ and $\mathcal{B}_{2}$, respectively. Let us denote the Lamé moduli of the material occupying the do main $\mathcal{B}_{\rho}$ by $\lambda^{(\rho)}\left(x_{1}, x_{2}\right)$ and $\mu^{(\rho)}\left(x_{1}, x_{2}\right)$, with $\left(x_{1}, x_{2}\right) \in S_{\rho}, \rho=1,2$.

Consider the problem of extension, bending and torsion of such a compound three dimensional beam, under the resultant forces and moments (20) acting on the ends. This problem has been trea ted in [23, Section 3.6], and the exact solution is expressed in terms of the solutions to some auxiliary plane strain problems. Let us de note by $u_{\alpha}^{(1)}, u_{\alpha}^{(2)}$ and $u_{\alpha}^{(3)}$ the solutions of the 3 plane strain prob lems $\mathcal{P}^{(1)}, \mathcal{P}^{(2)}$ and $\mathcal{P}^{(3)}$ respectively, formulated on the domain $\Sigma=S_{1} \cup S_{2} \cup \Gamma_{0}$ by

$$
\begin{aligned}
& \mathcal{P}^{(\gamma)}: t_{\beta \alpha, \beta}^{(\gamma)}+\left(\lambda^{(\rho)} x_{\gamma}\right)_{, \alpha} \quad 0 \text { in } S_{\rho}, t_{\beta \alpha}^{(\gamma)} n_{\beta}^{*} \quad \lambda^{(\rho)} x_{\gamma} n_{\alpha}^{*} \text { on } \Gamma_{\rho}, \\
& {\left[u_{\alpha}^{(\gamma)}\right]_{1} \quad\left[u_{\alpha}^{(\gamma)}\right]_{2}, \quad\left[t_{\beta \alpha}^{(\gamma)}\right]_{1} n_{\beta}^{0} \quad\left[t_{\beta \alpha}^{(\gamma)}\right]_{2} n_{\beta}^{0}+\left(\lambda^{(2)} \quad \lambda^{(1)}\right) x_{\gamma} n_{\alpha}^{0} \text { on } \Gamma_{0},} \\
& \mathcal{P}^{(3)}: t_{\beta \alpha, \beta}^{(3)}+\lambda_{, \alpha}^{(\rho)} \quad 0 \text { in } S_{\rho}, t_{\beta \alpha}^{(3)} n_{\beta}^{*} \quad \lambda^{(\rho)} n_{\alpha}^{*} \text { on } \Gamma_{\rho}, \\
& {\left[u_{\alpha}^{(3)}\right]_{1} \quad\left[u_{\alpha}^{(3)}\right]_{2}, \quad\left[t_{\beta \alpha}^{(3)}\right]_{1} n_{\beta}^{0} \quad\left[t_{\beta \alpha}^{(3)}\right]_{2} n_{\beta}^{0}+\left(\lambda^{(2)} \quad \lambda^{(1)}\right) n_{\alpha}^{0} \text { on } \Gamma_{0} .}
\end{aligned}
$$

We also introduce the function $\varphi\left(x_{1}, x_{2}\right)$ which is the solution of the boundary value problem

$$
\begin{aligned}
& \left(\mu^{(\rho)} \varphi_{, \alpha}\right)_{, \alpha} \quad \mu_{, 1}^{(\rho)} x_{2} \quad \mu_{, 2}^{(\rho)} x_{1} \text { in } S_{\rho}, \quad \frac{\partial \varphi}{\partial n^{*}} \quad x_{2} n_{1}^{*} \quad x_{1} n_{2}^{*} \text { on } \Gamma_{\rho}, \\
& {[\varphi]_{1} \quad[\varphi]_{2}, \quad \mu^{(1)}\left[\frac{\partial \varphi}{\partial n^{0}}\right]_{1} \quad \mu^{(2)}\left[\frac{\partial \varphi}{\partial n^{0}}\right]_{2}+\left(\mu^{(1)} \quad \mu^{(2)}\right)\left(x_{2} n_{1}^{0} \quad x_{1} n_{2}^{0}\right) \text { on } \Gamma_{0} .}
\end{aligned}
$$

Comparing the solution of the extension bending torsion problem in the direct approach given in Section 3.2 with the solution of the corresponding three dimensional problem presented in [23, Section 3.6], we deduce (in the same manner as in Section 4.1) the following expressions for the constitutive coefficients

$$
\begin{aligned}
& A_{3} \quad \sum_{\rho}^{2} \int_{S_{\rho}}\left(\lambda^{(\rho)}+2 \mu^{(\rho)}+\lambda^{(\rho)} u_{\gamma, \gamma}^{(3)}\right) \mathrm{d} x_{1} \mathrm{~d} x_{2}, \quad B_{13} \quad B_{23} \quad 0, \\
& B_{31} \quad \sum_{\rho}^{2} \int_{S_{\rho}} x_{2}\left(\lambda^{(\rho)}+2 \mu^{(\rho)}+\lambda^{(\rho)} u_{\gamma, \gamma}^{(3)}\right) \mathrm{d} x_{1} \mathrm{~d} x_{2}, \\
& B_{32} \sum_{\rho}^{2} \int_{S_{\rho}} x_{1}\left(\lambda^{(\rho)}+2 \mu^{(\rho)}+\lambda^{(\rho)} u_{\gamma, \gamma}^{(3)}\right) \mathrm{d} x_{1} \mathrm{~d} x_{2}, \\
& C_{1} \sum_{\rho}^{2} \int_{S_{\rho}} x_{2}\left[\left(\lambda^{(\rho)}+2 \mu^{(\rho)}\right) x_{2}+\lambda^{(\rho)} u_{\gamma, \gamma}^{(2)}\right] \mathrm{d} x_{1} \mathrm{~d} x_{2}, \\
& C_{2} \sum_{\rho}^{2} \int_{S_{\rho}} x_{1}\left[\left(\lambda^{(\rho)}+2 \mu^{(\rho)}\right) x_{1}+\lambda^{(\rho)} u_{\gamma, \gamma}^{(1)}\right] \mathrm{d} x_{1} \mathrm{~d} x_{2}, \\
& C_{12} \sum_{\rho}^{2} \int_{S_{\rho}} x_{1}\left[\left(\lambda^{(\rho)}+2 \mu^{(\rho)}\right) x_{2}+\lambda^{(\rho)} u_{\gamma, \gamma}^{(2)}\right] \mathrm{d} x_{1} \mathrm{~d} x_{2}, \\
& C_{3} \sum_{\rho}^{2} \int_{S_{\rho}} \mu^{(\rho)}\left[x_{1}\left(x_{1}+\varphi_{, 2}\right)+x_{2}\left(x_{2} \quad \varphi_{, 1}\right)\right] \mathrm{d} x_{1} \mathrm{~d} x_{2},
\end{aligned}
$$

where the functions $u_{\alpha}^{(k)}\left(x_{1}, x_{2}\right)$ are determined by (43) and $\varphi\left(x_{1}, x_{2}\right)$ is given by (44). 


\section{Remarks}

1. The above results (45) also hold when the distribution of the material in the beam is such that the separation curve $\Gamma_{0}$ is a closed curve included in $\Sigma$, see Fig. 2 b. In this case we have $\Gamma_{1}=\partial \Sigma, \Gamma_{2}=\emptyset, \partial S_{1}=\Gamma_{1} \cup \Gamma_{0}, \partial S_{2}=\Gamma_{0}$, and the boundary value problems (43), (44) keep the same forms.

2. The results of this section can be extended to the case when the beam $\mathcal{B}$ is composed of $n(n \geqslant 2)$ non homogeneous and isotro pic materials with different mechanical properties.

\section{Orthotropic and non-homogeneous materials}

Let us consider next beams and rods made of orthotropic and non homogeneous materials. The three dimensional constitutive equations for such materials are

$t_{11}^{*}=c_{11} e_{11}^{*}+c_{12} e_{22}^{*}+c_{13} e_{33}^{*}, t_{22}^{*}=c_{12} e_{11}^{*}+c_{22} e_{22}^{*}+c_{23} e_{33}^{*}$,

$t_{33}^{*}=c_{13} e_{11}^{*}+c_{23} e_{22}^{*}+c_{33} e_{33}^{*}, t_{23}^{*}=2 c_{44} e_{23}^{*}, \quad t_{31}^{*}=2 c_{55} e_{31}^{*}$,

$t_{12}^{*}=2 c_{66} e_{12}^{*}$,

where the constitutive coefficients $c_{i j}$ depend on $\left(x_{1}, x_{2}\right) \in \Sigma$.

Our aim is to determine the effective stiffness coefficients from the direct approach in terms of $c_{i j}\left(x_{1}, x_{2}\right)$. In this purpose, we con sider the extension, bending and torsion of the beam $\mathcal{B}$ due to the terminal loads (20).This three dimensional problem has been solved in [23, Section 4.11], with the help of some auxiliary plane strain problems defined on the domain $\Sigma$, which are recorded be low. We designate by $u_{\alpha}^{(k)}\left(x_{1}, x_{2}\right)$ the solutions of the plane strain problems $\mathcal{Q}^{(k)}, k \quad 1,2,3$, given by

$$
\begin{aligned}
& \mathcal{Q}^{(\gamma)}: t_{\beta \alpha, \beta}^{(\gamma)}+\left(c_{\alpha 3} x_{\gamma}\right)_{, \alpha} \quad 0 \quad \text { in } \Sigma, \quad t_{\beta \alpha}^{(\gamma)} n_{\beta}^{*} \quad c_{\alpha 3} x_{\gamma} n_{\alpha}^{*} \quad \text { on } \partial \Sigma \text {, } \\
& \mathcal{Q}^{(3)}: \quad t_{\beta \alpha, \beta}^{(3)}+c_{\alpha 3, \alpha} \quad 0 \quad \text { in } \Sigma, \quad t_{\beta \alpha}^{(3)} n_{\beta}^{*} \quad c_{\alpha 3} n_{\alpha}^{*} \quad \text { on } \partial \Sigma \text {. }
\end{aligned}
$$

The subscript $\alpha=1,2$ is not summed in the relations (47). The tor sion function $\varphi\left(x_{1}, x_{2}\right)$ is determined by the boundary value problem

$$
\begin{array}{lcccc}
\left(c_{55} \varphi_{, 1}\right)_{, 1}+\left(c_{44} \varphi_{, 2}\right)_{, 2} & c_{55,1} x_{2} & c_{44,2} x_{1} & \text { in } \Sigma, \\
c_{55} \varphi_{, 1} n_{1}^{*}+c_{44} \varphi_{, 2} n_{2}^{*} & c_{55} x_{2} n_{1}^{*} & c_{44} x_{1} n_{2}^{*} & \text { on } & \partial \Sigma .
\end{array}
$$

By identification of the three dimensional solution from [23, Sec tion 4.11], with the solution (17) (19) in the direct approach we get the following effective stiffness coefficients

$$
\begin{array}{lc}
A_{3} & \left\langle c_{33}+c_{13} u_{1,1}^{(3)}+c_{23} u_{2,2}^{(3)}\right\rangle, \\
B_{13} & B_{23} \quad 0, \\
B_{31} & \left\langle x_{2}\left(c_{33}+c_{13} u_{1,1}^{(3)}+c_{23} u_{2,2}^{(3)}\right)\right\rangle, \\
B_{32} & \left\langle x_{1}\left(c_{33}+c_{13} u_{1,1}^{(3)}+c_{23} u_{2,2}^{(3)}\right)\right\rangle, \\
C_{1} & \left\langle x_{2}\left(c_{33} x_{2}+c_{13} u_{1,1}^{(2)}+c_{23} u_{2,2}^{(2)}\right)\right\rangle, \\
C_{2} & \left\langle x_{1}\left(c_{33} x_{1}+c_{13} u_{1,1}^{(1)}+c_{23} u_{2,2}^{(1)}\right)\right\rangle, \\
C_{12} & \left\langle x_{1}\left(c_{33} x_{2}+c_{13} u_{1,1}^{(2)}+c_{23} u_{2,2}^{(2)}\right)\right\rangle, \\
C_{3} & \left\langle c_{44} x_{1}\left(x_{1}+\varphi_{, 2}\right)+c_{55} x_{2}\left(x_{2} \quad \varphi_{, 1}\right)\right\rangle .
\end{array}
$$

In view of the identifications (49) one can show that the fields $u, w_{\alpha}$, $\psi, \boldsymbol{N}$ and $\boldsymbol{M}$ corresponding to the solutions in the two approaches coincide.

Remark. This method can be applied also for beams composed of two different orthotropic materials. Using the notations introduced in the beginning of Section 5, we assume that the non homogeneous orthotropic material which occupies the domain $\mathcal{B}_{\rho}$ has the consti tutive coefficients $c_{i j}^{(\rho)}\left(x_{1}, x_{2}\right)$. If we employ the same procedure as in
Section 5 and compare with the results of [23, Section 4.11], then we obtain the following expressions for the effective stiffness coefficients

$$
\begin{array}{ll}
A_{3} \quad \sum_{\rho}^{2} \int_{S_{\rho}}\left(c_{33}^{(\rho)}+c_{13}^{(\rho)} u_{1,1}^{(3)}+c_{23}^{(\rho)} u_{2,2}^{(3)}\right) \mathrm{d} x_{1} \mathrm{~d} x_{2}, \quad B_{13} \quad B_{23} \quad 0, \\
B_{31} \quad \sum_{\rho}^{2} \int_{S_{\rho}} x_{2}\left(c_{33}^{(\rho)}+c_{13}^{(\rho)} u_{1,1}^{(3)}+c_{23}^{(\rho)} u_{2,2}^{(3)}\right) \mathrm{d} x_{1} \mathrm{~d} x_{2}, \\
B_{32} \quad \sum_{\rho}^{2} \int_{S_{\rho}} x_{1}\left(c_{33}^{(\rho)}+c_{13}^{(\rho)} u_{1,1}^{(3)}+c_{23}^{(\rho)} u_{2,2}^{(3)}\right) \mathrm{d} x_{1} \mathrm{~d} x_{2} \\
C_{1} \quad \sum_{\rho}^{2} \int_{S_{\rho}} x_{2}\left(c_{33}^{(\rho)} x_{2}+c_{13}^{(\rho)} u_{1,1}^{(2)}+c_{23}^{(\rho)} u_{2,2}^{(2)}\right) \mathrm{d} x_{1} \mathrm{~d} x_{2}, \\
C_{2} \quad \sum_{\rho}^{2} \int_{S_{\rho}} x_{1}\left(c_{33}^{(\rho)} x_{1}+c_{13}^{(\rho)} u_{1,1}^{(1)}+c_{23}^{(\rho)} u_{2,2}^{(1)}\right) \mathrm{d} x_{1} \mathrm{~d} x_{2}, \\
C_{12} \sum_{\rho}^{2} \int_{S_{\rho}} x_{1}\left(c_{33}^{(\rho)} x_{2}+c_{13}^{(\rho)} u_{1,1}^{(2)}+c_{23}^{(\rho)} u_{2,2}^{(2)}\right) \mathrm{d} x_{1} \mathrm{~d} x_{2}, \\
C_{3} \sum_{\rho}^{2} \int_{S_{\rho}}\left[c_{44}^{(\rho)} x_{1}\left(x_{1}+\varphi_{, 2}\right)+c_{55}^{(\rho)} x_{2}\left(x_{2} \quad \varphi_{, 1}\right)\right] \mathrm{d} x_{1} \mathrm{~d} x_{2},
\end{array}
$$

where $u_{\alpha}^{(k)}\left(x_{1}, x_{2}\right), k \quad 1,2,3$, are the solutions of the three plane strain problems

$$
\begin{aligned}
& t_{\beta \alpha, \beta}^{(\gamma)}+\left(c_{\alpha 3}^{(\rho)} x_{\gamma}\right)_{, \alpha} \quad 0 \quad \text { in } S_{\rho}, \quad t_{\beta \alpha}^{(\gamma)} n_{\beta}^{*} \quad c_{\alpha 3}^{(\rho)} x_{\gamma} n_{\alpha}^{*} \quad \text { on } \Gamma_{\rho}, \\
& {\left[u_{\alpha}^{(\gamma)}\right]_{1} \quad\left[u_{\alpha}^{(\gamma)}\right]_{2}, \quad\left[t_{\beta \alpha}^{(\gamma)}\right]_{1} n_{\beta}^{0} \quad\left[t_{\beta \alpha}^{(\gamma)}\right]_{2} n_{\beta}^{0}+\left(\begin{array}{ll}
c_{\alpha 3}^{(2)} & c_{\alpha 3}^{(1)}
\end{array}\right) x_{\gamma} n_{\alpha}^{0} \quad \text { on } \Gamma_{0},}
\end{aligned}
$$

$$
\begin{aligned}
& t_{\beta \alpha, \beta}^{(3)}+c_{\alpha 3, \alpha}^{(\rho)} \quad 0 \quad \text { in } S_{\rho}, \quad t_{\beta \alpha}^{(3)} n_{\beta}^{*} \quad c_{\alpha 3}^{(\rho)} n_{\alpha}^{*} \text { on } \Gamma_{\rho}, \\
& {\left[u_{\alpha}^{(3)}\right]_{1} \quad\left[u_{\alpha}^{(3)}\right]_{2}, \quad\left[t_{\beta \alpha}^{(3)}\right]_{1} n_{\beta}^{0} \quad\left[t_{\beta \alpha}^{(3)}\right]_{2} n_{\beta}^{0}+\left(\begin{array}{lll}
c_{\alpha 3}^{(2)} & c_{\alpha 3}^{(1)}
\end{array}\right) n_{\alpha}^{0} \quad \text { on } \Gamma_{0} .}
\end{aligned}
$$

In the relations (51) and (52) the subscript $\alpha=1,2$ is not summed. The torsion function $\varphi\left(x_{1}, x_{2}\right)$ appearing in (50) is the solution of the following boundary value problem

$$
\begin{aligned}
& \left(c_{55}^{(\rho)} \varphi_{, 1}\right)_{, 1}+\left(c_{44}^{(\rho)} \varphi_{, 2}\right)_{, 2} \quad c_{55,1}^{(\rho)} x_{2} \quad c_{44,2}^{(\rho)} x_{1} \text { in } S_{\rho}, \\
& c_{55}^{(\rho)} \varphi_{, 1} n_{1}^{*}+c_{44}^{(\rho)} \varphi_{, 2} n_{2}^{*} \quad c_{55}^{(\rho)} x_{2} n_{1}^{*} \quad c_{44}^{(\rho)} x_{1} n_{2}^{*} \quad \text { on } \Gamma_{\rho}, \quad[\varphi]_{1} \quad[\varphi]_{2} \text { on } \Gamma_{0} \text {, } \\
& {\left[c_{55}^{(1)} \varphi_{, 1} n_{1}^{0}+c_{44}^{(1)} \varphi_{, 2} n_{2}^{0}\right]_{1} \quad\left[c_{55}^{(2)} \varphi_{, 1} n_{1}^{0}+c_{44}^{(2)} \varphi_{, 2} n_{2}^{0}\right]_{2}} \\
& +\left(\begin{array}{ll}
c_{55}^{(1)} & c_{55}^{(2)}
\end{array}\right) x_{2} n_{1}^{0} \quad\left(\begin{array}{ll}
c_{44}^{(1)} & c_{44}^{(2)}
\end{array}\right) x_{1} n_{2}^{0} \text { on } \Gamma_{0} .
\end{aligned}
$$

The relations (50) for the constitutive coefficients are valid also in the case when $\Gamma_{0}$ is a closed curve included in $\Sigma$. Moreover, these formulas can be extended to the case of beams composed of $n$ dif ferent orthotropic materials $(n \geqslant 2)$.

\subsection{Transverse shear stiffness}

To determine the transverse shear stiffness coefficients $A_{1}, A_{2}$ and $A_{12}$ for orthotropic non homogeneous rods, we consider the problem of shear vibrations of rectangular rods formulated in Sec tion 4.2. Assume that $\rho^{*}$ has a symmetrical distribution in the $x_{1}$ direction: $\rho^{*}\left(x_{1}, x_{2}\right)=\rho^{*}\left(x_{1}, x_{2}\right)$.

We search for a solution in the form (31). Then the boundary conditions (30) are satisfied and the equations of motion reduce to

$c_{55}\left(x_{1}, x_{2}\right) \frac{\pi}{a} \cos \left(\frac{\pi}{a} x_{1}\right) \quad \omega^{2} \int_{a / 2}^{x_{1}} \rho^{*}\left(x_{1}, x_{2}\right) \sin \left(\frac{\pi}{a} x_{1}\right) \mathrm{d} x_{1}$ 
Inserting here the relation (33) and integrating over $\Sigma$ we find the lowest natural frequency

$\omega^{2} \quad\left(\frac{\pi}{a}\right)^{2} \frac{\left\langle c_{55}\left(x_{1}, x_{2}\right)\right\rangle}{\left\langle\rho^{*}\left(\alpha, x_{2}\right)\right\rangle}$.

On the other hand, we solve the same problem by the direct ap proach and we find the rod's natural frequency $\bar{\omega}$ given by (37). We identify $\omega \quad \bar{\omega}$ and from relations (37) and (54) we obtain

$A_{1} \quad k \frac{\left\langle c_{55}\right\rangle\left\langle\rho^{*} x_{1}^{2}\right\rangle \operatorname{Area}(\Sigma)}{\left\langle\rho^{*}\left(\alpha, x_{2}\right)\right\rangle\left\langle x_{1}^{2}\right\rangle}, A_{12} \quad 0$.

To determine $A_{2}$, one can proceed analogously in the $x_{2}$ direction.

\section{Remarks}

1. If we admit the approximation (40) then we deduce

$$
\begin{aligned}
& A_{1} \quad k\left\langle c_{55}\right\rangle \frac{\left\langle\rho^{*} x_{1}^{2}\right\rangle \operatorname{Area}(\Sigma)}{\left\langle\rho^{*}\right\rangle\left\langle x_{1}^{2}\right\rangle}, \\
& A_{2} \quad k\left\langle c_{44}\right\rangle \frac{\left\langle\rho^{*} x_{2}^{2}\right\rangle \operatorname{Area}(\Sigma)}{\left\langle\rho^{*}\right\rangle\left\langle x_{2}^{2}\right\rangle} .
\end{aligned}
$$

where $\frac{\pi^{2}}{12}$ stands for the value of the factor $k$. In most cases, these formulas are applicable for orthotropic non homogeneous rods with arbitrary cross section properties (not necessarily rectan gular or symmetrical).

2. Consider the case of non homogeneous rods composed of two different orthotropic materials: in the region $\mathcal{B}_{\gamma}$ of the body we have the mass density $\rho^{(\gamma)}\left(x_{1}, x_{2}\right)$ and the constitutive coef ficients $c_{i j}^{(\gamma)}\left(x_{1}, x_{2}\right), \gamma$ 1,2. Eqs. (55) and (56) for transverse shear stiffness coefficients remain valid also in this case, with the specifications

$$
\begin{aligned}
& \left\langle c_{i j}\right\rangle \quad \sum_{\gamma=1}^{2} \int_{S_{\gamma}} c_{i j}^{(\gamma)} \mathrm{d} x_{1} \mathrm{~d} x_{2},\left\langle\rho^{*}\right\rangle \quad \sum_{\gamma=1}^{2} \int_{S_{\gamma}} \rho^{(\gamma)} \mathrm{d} x_{1} \mathrm{~d} x_{2}, \\
& \left\langle\rho^{*} x_{\alpha}^{2}\right\rangle \quad \sum_{\gamma=1}^{2} \int_{S_{\gamma}} \rho^{(\gamma)} x_{\alpha}^{2} \mathrm{~d} x_{1} \mathrm{~d} x_{2} .
\end{aligned}
$$

The extension of formulas (56) and (57) to the case of rods com posed of $n$ orthotropic materials is also possible.

\section{Special cases and examples}

\subsection{Non homogeneous rods with constant Poisson ratio}

Let us consider the case when the rod is made of an isotropic material with constant Poisson ratio $v$. The Young's modulus $E$ is an arbitrary function of $\left(x_{1}, x_{2}\right)$ and the shape of cross section $\Sigma$ is arbitrary. This type of material is of practical interest and it has been studied in many works, see e.g. [41]. In this case the solu tions $u_{\alpha}^{(k)}\left(x_{1}, x_{2}\right)$ of the problems $\mathcal{D}^{(k)}, k \quad 1,2,3$, defined by (21) have a simple form

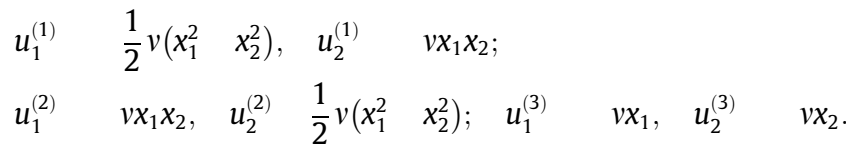

Then, from (29) we obtain the following expressions for the effec tive stiffness coefficients
$A_{3}\left\langle E\left(x_{1}, x_{2}\right)\right\rangle, C_{12}$
$\left\langle x_{1} x_{2} E\left(x_{1}, x_{2}\right)\right\rangle, C_{1}$
$\left\langle x_{2}^{2} E\left(x_{1}, x_{2}\right)\right\rangle$,
$C_{2}\left\langle x_{1}^{2} E\left(x_{1}, x_{2}\right)\right\rangle, B_{31} \quad\left\langle x_{2} E\left(x_{1}, x_{2}\right)\right\rangle, B_{32} \quad\left\langle x_{1} E\left(x_{1}, x_{2}\right)\right\rangle, B_{\alpha 3} \quad 0$.

The constitutive coefficients $C_{3}, A_{1}, A_{2}$ and $A_{12}$ keep the same form as in the general case, given by $(29)_{1}$ and (38).
Remark. In the case of a homogeneous isotropic rod, i.e. when $E$ is also constant, from (59) and (5) we obtain the well known formulas

$A_{3} \quad E \operatorname{Area}(\Sigma), \quad C_{1} \quad E\left\langle x_{2}^{2}\right\rangle, \quad C_{2} \quad E\left\langle x_{1}^{2}\right\rangle, \quad C_{12} \quad 0$, $B_{\alpha 3} \quad B_{3 \alpha} \quad 0$

In view of $(29)_{1}$ the torsional rigidity $C_{3}$ for simply connected cross sections is given by

$C_{3} 2 \mu\left\langle\phi\left(x_{1}, x_{2}\right)\right\rangle$ with $\Delta \phi \quad 2$ in $\Sigma, \quad \phi \quad 0$ on $\partial \Sigma$.

The effective transverse shear coefficients are given by (39). The above expressions of the effective stiffness coefficients for homoge neous and isotropic directed curves have been presented in [20,21].

\subsection{Circular rod composed of two materials}

For rods composed of two different isotropic and non homoge neous materials we use the notations and developments of Section 5 . The cross section of the rod is decomposed as $\Sigma \quad S_{1} \cup \bar{S}_{2}$, where $S_{1} \quad\left\{\left(x_{1}, x_{2}\right) \mid a^{2}<x_{1}^{2}+x_{2}^{2}<b^{2}\right\}$ and $S_{2} \quad\left\{\left(x_{1}, x_{2}\right) \mid x_{1}^{2}+x_{2}^{2}<a^{2}\right\}$. The first material occupies the region $S_{1} \times(0, l)$ and has the Lamé moduli

$$
\begin{aligned}
& \lambda^{(1)}\left(x_{1}, x_{2}\right) \quad \lambda_{0} r^{m}, \quad \mu^{(1)}\left(x_{1}, x_{2}\right) \quad \mu_{0} r^{m}, \\
& r \quad \sqrt{x_{1}^{2}+x_{2}^{2},\left(x_{1}, x_{2}\right) \in S_{1}}
\end{aligned}
$$

where $m>0, \lambda_{0}$ and $\mu_{0}$ are constants. This kind of inhomogeneity has been investigated in many works, e.g. [41,42]. We denote by $v_{0} \frac{\lambda_{0}}{2\left(\lambda_{0}+\mu_{0}\right)}$ and $E_{0} \frac{\mu_{0}\left(3 \lambda_{0}+2 \mu_{0}\right)}{\lambda_{0}+\mu_{0}}$. The second material occupies the re gion $S_{2} \times(0, l)$ and its elastic properties are described by

$$
\begin{aligned}
& E^{(2)}\left(x_{1}, x_{2}\right) \quad E(r), \quad v^{(2)}\left(x_{1}, x_{2}\right) \quad v_{0} \text { (constant), } \\
& \left(x_{1}, x_{2}\right) \in S_{2},
\end{aligned}
$$

where $E(r)$ is an arbitrary given function of $r$.

In order to use the results presented in Section 5 we have to solve the plane strain problems $\mathcal{P}^{(k)}$ given by (43) and the bound ary value problem (44) for the torsion function. In our case, we ob serve that these problems admit the following solutions

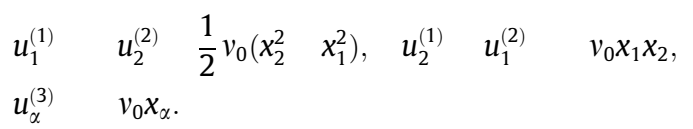

Inserting these functions into the general results (45) we find the effective stiffness coefficients for this compound rod

$$
\begin{aligned}
& C_{3} \frac{\pi}{1+v_{0}} \int_{0}^{a} r^{3} E(r) \mathrm{d} r+2 \pi \mu_{0} d_{m}, \quad A_{3} \quad 2 \pi\left(\int_{0}^{a} r E(r) \mathrm{d} r+E_{0} c_{m}\right), \\
& C_{1} \quad C_{2} \quad \pi\left(\int_{0}^{a} r^{3} E(r) \mathrm{d} r+E_{0} d_{m}\right), \quad C_{12} \quad 0, \quad B_{3 \alpha} \quad B_{\alpha 3} \quad 0,
\end{aligned}
$$

where we have denoted by $c_{m}$ and $d_{m}$ the expressions

$$
c_{m}\left\{\begin{array}{ll}
\frac{a^{2} m b^{2} m}{m 2} & \text { for } m \neq 2 \\
\log (b / a) & \text { for } m \quad 2
\end{array}, \quad d_{m}\left\{\begin{array}{ll}
\frac{a^{4} m b^{4}}{m} & \text { for } m \neq 4 \\
\log (b / a) & \text { for } m \quad 4
\end{array} .\right.\right.
$$

Let us find also the transverse shear stiffness coefficients $A_{1}$ and $A_{2}$. Assume that the mass density function $\rho^{*}\left(x_{1}, x_{2}\right)$ is given by

$$
\rho^{*}\left(x_{1}, x_{2}\right) \quad\left\{\begin{array}{cc}
\rho_{0}^{*} r^{m} & \text { for }\left(x_{1}, x_{2}\right) \in S_{1} \\
\rho(r) & \text { for }\left(x_{1}, x_{2}\right) \in S_{2}
\end{array},\right.
$$

where $\rho_{0}^{*}>0$ is a constant and $\rho(r)$ is an arbitrary function. Then, using the results (56), (57) specialized for isotropic materials we find the expressions 
$A_{1} \quad A_{2} \quad \frac{\pi^{3}}{6 b^{2}}\left(\int_{0}^{a} \frac{r E(r)}{1+v_{0}} \mathrm{~d} r+2 \mu_{0} c_{m}\right)\left(\int_{0}^{a} r^{3} \rho(r) \mathrm{d} r+\rho_{0}^{*} d_{m}\right)$

$$
\times\left(\int_{0}^{a} r \rho(r) \mathrm{d} r+\rho_{0}^{*} c_{m}\right)^{1} .
$$

\subsection{Orthotropic circular rod}

Let us consider an orthotropic rod with cross section $\Sigma\left\{\left(x_{1}, x_{2}\right) \mid x_{1}^{2}+x_{2}^{2}<a^{2}\right\}$. We assume that the constitutive coeffi cients satisfy

$c_{i j} \quad c_{i j}^{*} e^{\sigma r}, \quad r \quad \sqrt{x_{1}^{2}+x_{2}^{2}}$,

where $\sigma>0$ and $c_{i j}^{*}$ are constants. Let us introduce the notations

$$
\begin{aligned}
& E_{0} \quad E_{0}^{*} e^{\sigma r}, \quad E_{0}^{*} \quad c_{33}^{*} \quad c_{13}^{*} v_{1}^{*} \quad c_{23}^{*} v_{2}^{*}, \\
& v_{1}^{*} \quad \frac{c_{13}^{*} c_{22}^{*} \quad c_{23}^{*} c_{12}^{*}}{\delta_{1}^{*}}, \quad v_{2}^{*} \quad \frac{c_{23}^{*} c_{11}^{*} c_{13}^{*} c_{12}^{*}}{\delta_{1}^{*}}, \quad \delta_{1}^{*} \quad c_{11}^{*} c_{22}^{*} \quad\left(c_{12}^{*}\right)^{2} .
\end{aligned}
$$

The solutions $u_{\alpha}^{(k)}\left(x_{1}, x_{2}\right)$ of the problems $\mathcal{Q}^{(k)}$ given by (47) are in this case

$$
\begin{aligned}
& u_{1}^{(1)} \quad \frac{1}{2}\left(v_{1}^{*} x_{1}^{2} \quad v_{2}^{*} x_{2}^{2}\right), \quad u_{2}^{(1)} \quad v_{2}^{*} x_{1} x_{2} ; \\
& u_{1}^{(2)} \quad v_{1}^{*} x_{1} x_{2}, \quad u_{2}^{(2)} \quad \frac{1}{2}\left(v_{1}^{*} x_{1}^{2} \quad v_{2}^{*} x_{2}^{2}\right) \text {; } \\
& u_{1}^{(3)} \quad v_{1}^{*} x_{1}, \quad u_{2}^{(3)} \quad v_{2}^{*} x_{2} \text {, }
\end{aligned}
$$

while the torsion function $\varphi\left(x_{1}, x_{2}\right)$ which solves the boundary va lue problem (48) is

$\varphi\left(x_{1}, x_{2}\right) \quad \frac{c_{55}^{*} \quad c_{44}^{*}}{c_{44}^{*}+c_{55}^{*}} x_{1} x_{2}$.

Thus, in view of (49) and (69) and (70) we find the effective stiffness coefficients

$$
\begin{aligned}
& C_{3} \frac{4 \pi c_{44}^{*} C_{55}^{*}}{c_{44}^{*}+c_{55}^{*}} \int_{0}^{a} r^{3} e^{\sigma r} \mathrm{~d} r, \quad A_{3} \quad 2 \pi E_{0}^{*} \int_{0}^{a} r e^{\sigma r} \mathrm{~d} r, \\
& \begin{array}{llllllll}
C_{1} & C_{2} & \pi E_{0}^{*} \int_{0}^{a} r^{3} e^{\sigma r} \mathrm{~d} r, & C_{12} & 0, & B_{3 \alpha} & B_{\alpha 3} & 0 .
\end{array}
\end{aligned}
$$

Assume that the mass density of the rod is of the form $\rho^{*}\left(x_{1}, x_{2}\right) \quad \rho_{0}^{*} e^{\sigma r}$, where $\rho_{0}^{*}>0$ is constant. Then, from relations (56) we obtain the effective transverse shear stiffness coefficients

$A_{1} \quad \frac{\pi^{3}}{3} \frac{c_{55}^{*}}{a^{2}} \int_{0}^{a} r^{3} e^{\sigma r} \mathrm{~d} r, \quad A_{2} \quad \frac{\pi^{3}}{3} \frac{c_{44}^{*}}{a^{2}} \int_{0}^{a} r^{3} e^{\sigma r} \mathrm{~d} r$.

\section{Functionally graded beams made of metal foams}

\subsection{Distribution of the material properties}

The mechanical properties of cellular solids have been pre sented in the books $[25,43]$. In this section we analyze rectangular beams made of metal foams.

The cross section domain is given by $\Sigma \quad\left\{\left(x_{1}, x_{2}\right) \mid x_{1} \in\left(\frac{h}{2}, \frac{h}{2}\right), x_{2} \in\left(\frac{b}{2}, \frac{b}{2}\right)\right\}$. We consider that the porous material is functionally graded in the $x_{1}$ direction, such that the mass density $\rho$ of the foam is given as a function of $x_{1}$ by the power law

$\rho\left(x_{1}\right) \quad \rho_{m}+\left(\begin{array}{ll}\rho_{s} & \rho_{m}\end{array}\right)\left(\frac{2\left|x_{1}\right|}{h}\right)^{N}$,

where $\rho_{s}$ is the density of the bulk (matrix) material, $\rho_{m}$ is the min imum value of the density of the foam, and $N$ is an exponent. This type of functionally graded porous materials has been studied in the case of plates in $[43,44]$. To express the Young modulus $E$ of the foam we use the formula indicated by Gibson and Ashby [25]

$E\left(x_{1}\right) \quad E_{s}\left(\frac{\rho\left(x_{1}\right)}{\rho_{s}}\right)^{\kappa}$,

where $E_{s}$ is the Young modulus of the bulk material. In what follows, we consider closed cell aluminum foams, for which the exponent $\kappa$ is given by $\kappa=2$, and the Poisson ratio is assumed to be constant with the value $v=0.3$ [25]. Let us denote by $G_{s} \frac{E_{s}}{2(1+v)}$ the shear modulus of the bulk material. The variations of $\rho$ and $E$ as functions of $x_{1}$, as given in (73) and (74), are depicted in Fig. 3 for several val ues of the exponent $N$.

Let us calculate the effective stiffness coefficients for this func tionally graded porous beam. Since the Poisson ratio is assumed constant, we can use the relation (59), in conjunction with (73) and (74), to derive the extensional and bending stiffness coefficients

$$
\begin{aligned}
& A_{3} \quad b h E_{s}\left[r^{2}+\frac{2}{N+1} r(1 \quad r)+\frac{1}{2 N+1}\left(\begin{array}{ll}
1 & r
\end{array}\right)^{2}\right], \quad B_{3 \alpha} \quad B_{\alpha 3} \quad 0, \\
& C_{1} \quad \frac{b^{3} h}{12} E_{s}\left[r^{2}+\frac{2}{N+1} r(1 \quad r)+\frac{1}{2 N+1}\left(\begin{array}{ll}
1 & r
\end{array}\right)^{2}\right], \quad C_{12} \quad 0, \\
& C_{2} \quad \frac{b h^{3}}{12} E_{s}\left[r^{2}+\frac{6}{N+3} r(1 \quad r)+\frac{3}{2 N+3}\left(\begin{array}{ll}
1 & r)^{2}
\end{array}\right]\right. \text {, }
\end{aligned}
$$

where we denote by $r$ the ratio $r \quad \frac{\rho_{m}}{\rho_{c}}$. The effective shear stiffness can be calculated from the relations (38). We insert the expression for $\rho$ from (73) into (38) and obtain

$$
\begin{aligned}
& A_{2} \quad k b h G_{s}\left[r^{2}+\frac{2}{N+1} r\left(\begin{array}{ll}
1 & r
\end{array}\right)+\frac{1}{2 N+1}\left(\begin{array}{ll}
1 & r
\end{array}\right)^{2}\right] \text {, } \\
& A_{1} \quad k b h G_{s} \frac{(b h) \rho_{s}}{\left\langle\rho\left(\alpha\left(x_{1}\right)\right)\right\rangle}\left[r+\frac{3}{N+3}\left(\begin{array}{ll}
1 & r)
\end{array}\right]\right. \\
& \times\left[r^{2}+\frac{2}{N+1} r(1 \quad r)+\frac{1}{2 N+1}\left(\begin{array}{ll}
1 & r
\end{array}\right)^{2}\right],
\end{aligned}
$$

where, according to (33), $\left\langle\rho\left(\alpha\left(x_{1}\right)\right)\right\rangle$ is given by

$$
\left\langle\rho\left(\alpha\left(x_{1}\right)\right)\right\rangle \quad \frac{\pi b}{h} \int_{\frac{h}{2}}^{\frac{h}{2}}\left(\cos \frac{\pi x_{1}}{h}\right)^{1} \int_{\frac{h}{2}}^{x_{1}} \rho(\zeta) \sin \frac{\pi \zeta}{h} \mathrm{~d} \zeta \mathrm{d} x_{1} .
$$

Using the expression (73) in (77) and making some mathematical calculations, we get

$$
\left\langle\rho\left(\alpha\left(x_{1}\right)\right)\right\rangle \quad b h \rho_{s}\left[r+\frac{1 \quad r}{N+1}+\left(\begin{array}{ll}
1 & r
\end{array}\right) J_{N}\right],
$$

where we have denoted by

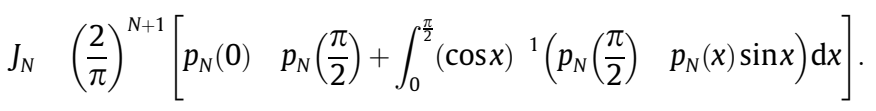

In the last relation, the polynomial function $p_{N}(x)$ is given by

$$
\begin{aligned}
& p_{N}(x) \quad N x^{N 1} \quad N\left(\begin{array}{lll}
N & 1
\end{array}\right)\left(\begin{array}{lll}
N & 2
\end{array}\right) x^{N}{ }^{3}+N\left(\begin{array}{ll}
N & 1
\end{array}\right) \quad\left(\begin{array}{ll}
N & 4
\end{array}\right) x^{N} 5 \\
& \sum_{i=0}^{m}(1)^{i} N(N \quad 1) \quad(N \quad 2 i) x^{N} 2 i 1, \quad p_{1}(x) \quad 1, p_{2}(x) \quad 2 x \text {, }
\end{aligned}
$$

where $m \quad\left[\frac{N 1}{2}\right]$ is the greatest integer not exceeding $\frac{N 1}{2}$.

Finally, if we substitute (78) into $(76)_{2}$ we find

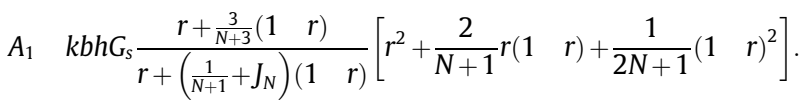

The formula (79) represent the 'exact' expression for the effective shear stiffness, calculated on the basis of (38). On the other hand, if we employ the 'approximate' relation (41) instead of (38), then we deduce the following simplified (approximate) expression for $A_{1}$ 

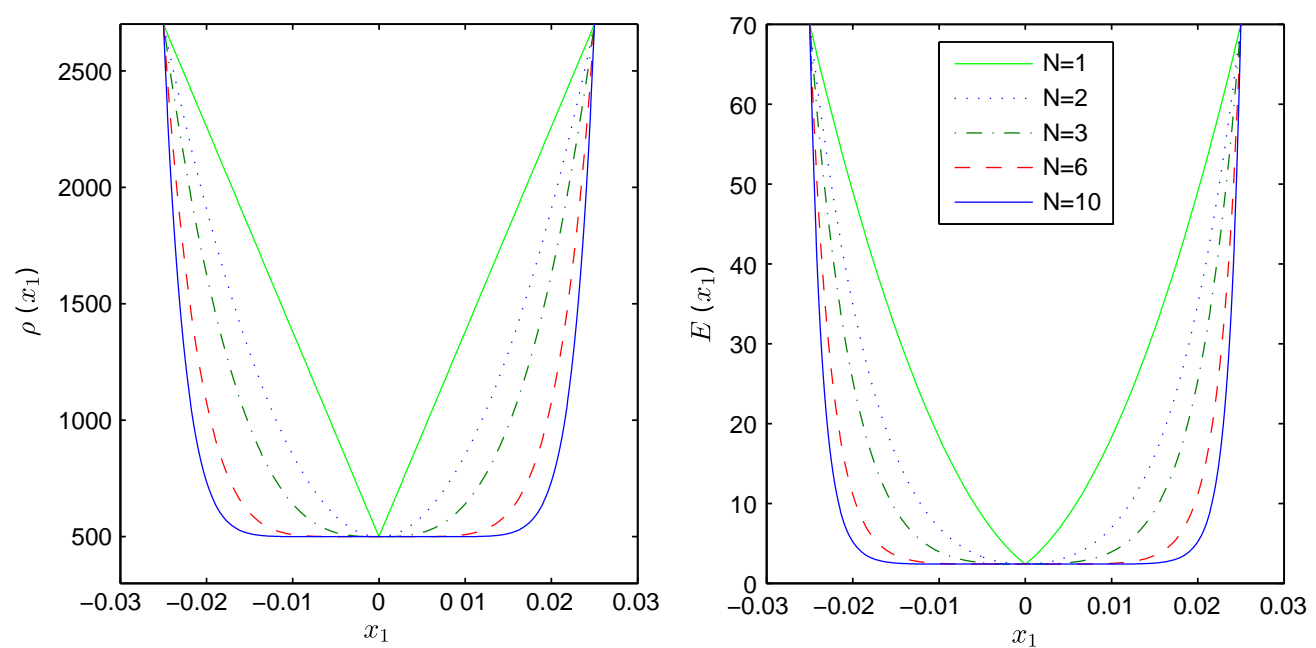

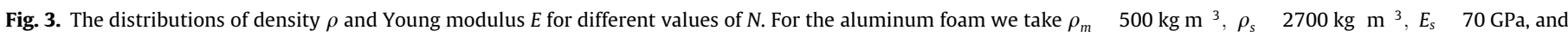
the thickness $h=0.05 \mathrm{~m}$.

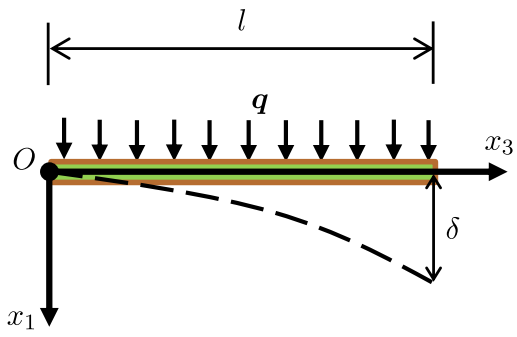

(a)

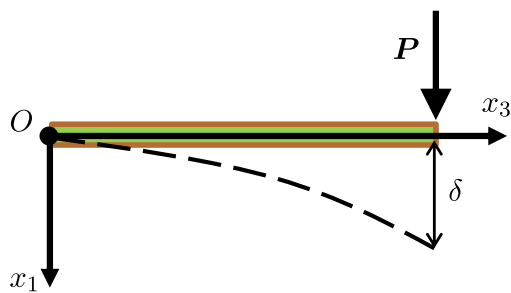

(b)

Fig. 4. (a) Cantilever beam with uniform distributed load $q$. (b) Cantilever beam with concentrated end force $P$.

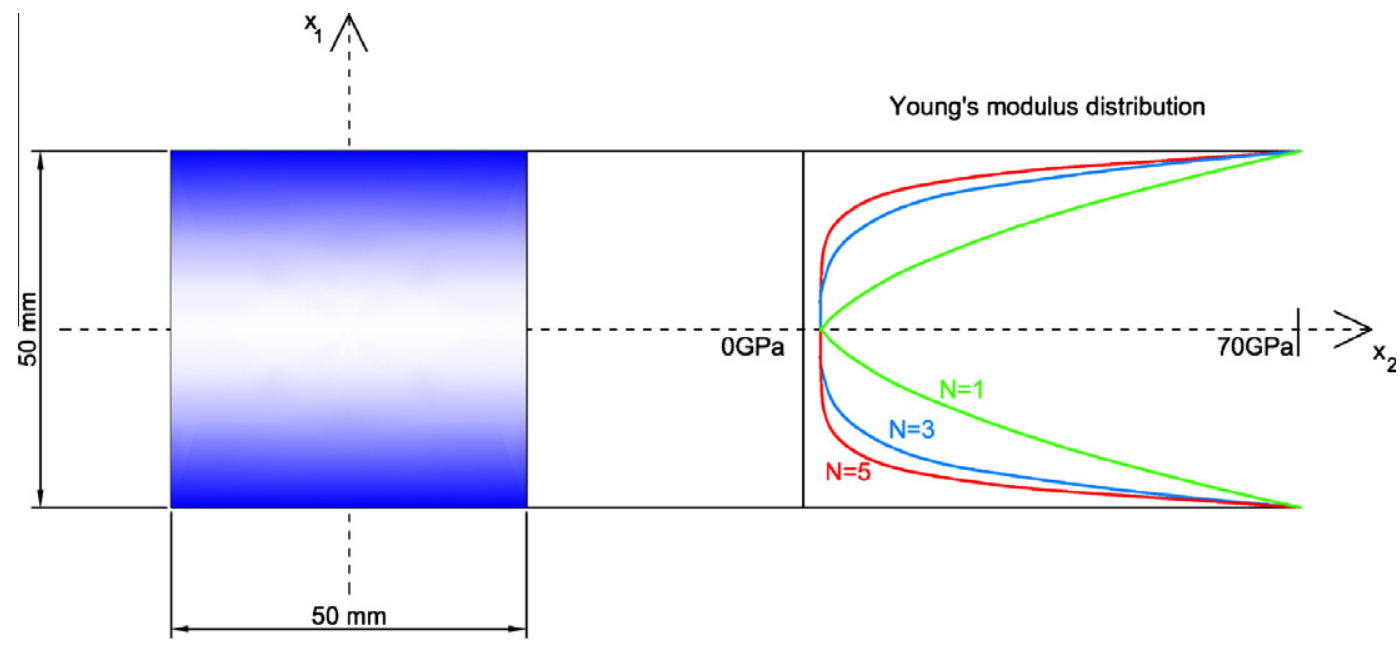

Fig. 5. Cross-section of the FGM beam and distribution of Young's modulus.

$\widetilde{A}_{1} \quad k b h G_{s}$

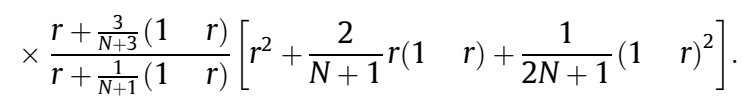

Let us use the effective stiffness coefficients for FGM porous beams determined previously to solve some bending problems and compare the analytical solutions with the results obtained by a fi nite element analysis.

\subsection{Cantilever beams}

Consider a cantilever beam made of functionally graded closed cell aluminum foam subject to bending and shear under 
Table 1

Comparison of results for cantilever FGM beam with uniform load.

\begin{tabular}{|c|c|c|c|c|c|c|c|c|c|c|}
\hline$N$ & 1 & 2 & 3 & 4 & 5 & 6 & 7 & 8 & 9 & 10 \\
\hline$\delta_{\mathrm{FEM}}(\mathrm{mm})$ & 26.316 & 34.825 & 42.818 & 50.406 & 57.649 & 64.581 & 71.223 & 77.591 & 83.699 & 89.557 \\
\hline$\delta_{\text {exact }}(\mathrm{mm})$ & 26.129 & 34.471 & 42.410 & 50.025 & 57.358 & 64.435 & 71.276 & 77.896 & 84.308 & 90.523 \\
\hline$\delta_{\text {approx }}(\mathrm{mm})$ & 26.097 & 34.407 & 42.317 & 49.907 & 57.218 & 64.276 & 71.100 & 77.705 & 84.105 & 90.309 \\
\hline$\Delta(\%)$ & 0.716 & 1.027 & 0.962 & 0.762 & 0.507 & 0.227 & -0.074 & -0.393 & -0.728 & -1.079 \\
\hline
\end{tabular}

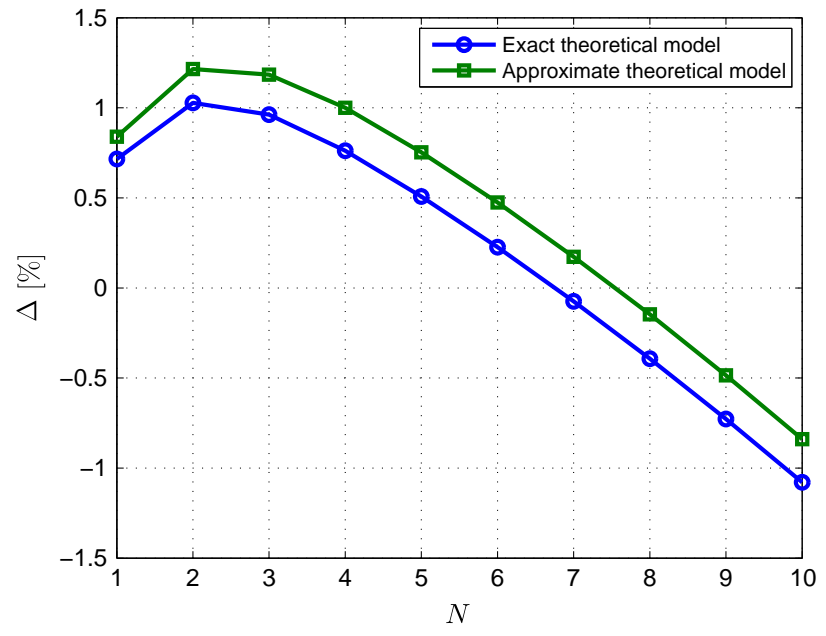

Fig. 6. Error $\Delta$ in terms of the exponent $N$, for the maximum deflection of a cantilever FGM beam with uniform load.

the following loads: (a) uniformly distributed force $q$ acting in the $x_{1}$ direction; or (b) concentrated end force $P$ acting in the $x_{1}$ direc tion. We denote by $l$ the length of the beam (see Fig. 4).

The analytical solutions of these problems can easily be derived from the one dimensional governing differential equations of $\mathrm{di}$ rected rods presented in Section 3. For the maximum deflection $\delta$ of the beam we obtain the well known relations

$\delta \quad \frac{q l^{2}}{2}\left(\frac{1}{A_{1}}+\frac{l^{2}}{4 C_{2}}\right) \quad$ for uniformly distributed force $q$,

$\delta \quad P l\left(\frac{1}{A_{1}}+\frac{l^{2}}{3 C_{2}}\right) \quad$ for concentrated end force $P$,

where the values of the effective shear stiffness $A_{1}$ and bending stiffness $C_{2}$ for FGM porous beams are given by (79) (or the approx imate form (80)) and (75), respectively. The theoretical predictions (81) will be compared with numerical solutions obtained by the fi nite element method.

The cross section of the beam has the dimensions $h=50 \mathrm{~mm}$ and $b=50 \mathrm{~mm}$ (see Fig. 5), the length is $l=1 \mathrm{~m}$, and the closed cell aluminum foam is characterized by the material parameters $\rho_{m} \quad 500 \mathrm{~kg} \mathrm{~m}^{3}, \rho_{s} \quad 2700 \mathrm{~kg} \mathrm{~m}^{3}, E_{s} \quad 70 \mathrm{GPa}$. We have calcu lated the maximum deflection of the beam numerically, using the software ABAQUS. To describe its functionally graded structure, the beam domain has been divided into layers orthogonal to the $x_{1}$ direction. Each layer is assumed to have constant material param eters $E$ and $\rho$, which satisfy the power laws (73) and (74) stepwise.

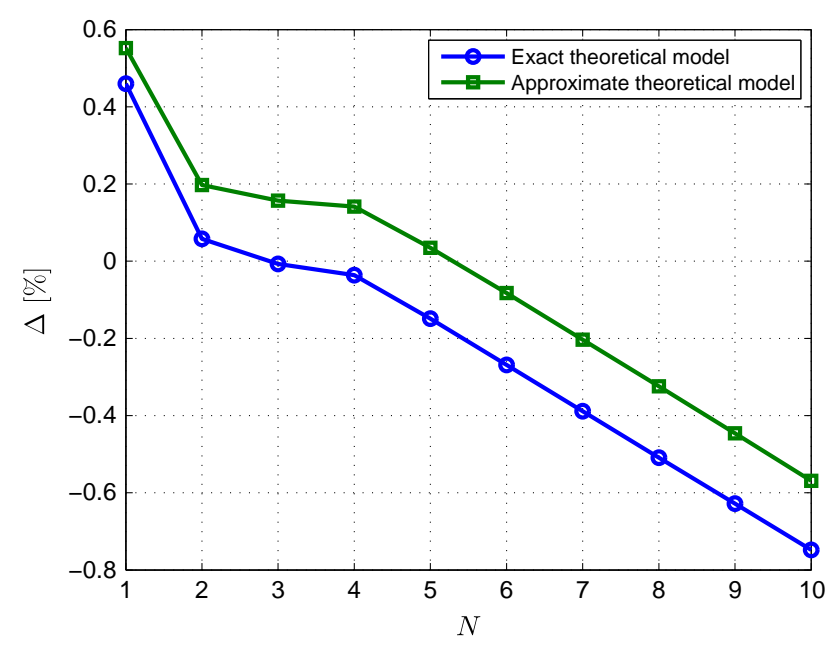

Fig. 7. Error $\Delta$ in terms of the exponent $N$, for the maximum deflection of a cantilever FGM beam with end load.

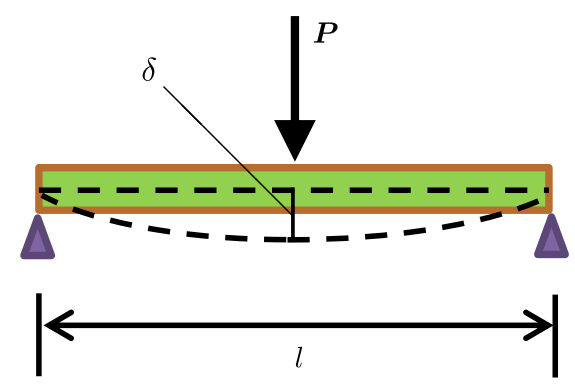

Fig. 8. Three-point bending of a FGM beam.

For the problems presented here a number of 64 or 128 layers is sufficient. The calculation has been performed using 3D shell ele ments and very dense mesh. The finite elements have been taken square, with one element per layer thickness.

We denote by $\delta_{\mathrm{FEM}}$ the maximum deflection calculated by finite element analysis, let $\delta_{\text {exact }}$ be the theoretical value of the maximum deflection given by (81) with the exact formula (79) for $A_{1}$, and $\delta_{\text {approx }}$ be the theoretical value given by (81) with the approximate formula (80). We calculate the relative error $\Delta$ by the relation

$$
\Delta \frac{\delta_{\mathrm{FEM}} \delta_{\text {exact }}}{\min \left(\delta_{\mathrm{FEM}} ; \delta_{\text {exact }}\right)} .
$$

Table 2

Comparison of results for cantilever FGM beam with concentrated end load.

\begin{tabular}{|c|c|c|c|c|c|c|c|c|c|c|}
\hline$N$ & 1 & 2 & 3 & 4 & 5 & 6 & 7 & 8 & 9 & 10 \\
\hline$\delta_{\mathrm{FEM}}(\mathrm{mm})$ & 69.920 & 91.856 & 112.927 & 133.158 & 152.499 & 171.107 & 189.044 & 206.355 & 223.076 & 239.238 \\
\hline$\delta_{\text {exact }}(\mathrm{mm})$ & 69.600 & 91.803 & 112.935 & 133.206 & 152.726 & 171.567 & 189.779 & 207.405 & 224.477 & 241.027 \\
\hline$\delta_{\text {approx }}(\mathrm{mm})$ & 69.536 & 91.675 & 112.750 & 132.970 & 152.446 & 171.248 & 189.428 & 207.024 & 224.071 & 240.599 \\
\hline$\Delta(\%)$ & 0.460 & 0.058 & -0.007 & -0.036 & -0.149 & -0.269 & -0.389 & -0.509 & -0.628 & -0.748 \\
\hline
\end{tabular}


Table 3

Comparison of results for FGM beam in three-point bending.

\begin{tabular}{|c|c|c|c|c|c|c|c|c|c|c|}
\hline$N$ & 1 & 2 & 3 & 4 & 5 & 6 & 7 & 8 & 9 & 10 \\
\hline$\delta_{\mathrm{FEM}}(\mathrm{mm})$ & 4.476 & 5.972 & 7.356 & 8.660 & 9.900 & 11.088 & 12.230 & 13.329 & 14.389 & 15.412 \\
\hline$\delta_{\text {exact }}(\mathrm{mm})$ & 4.393 & 5.804 & 7.147 & 8.435 & 9.674 & 10.870 & 12.024 & 13.141 & 14.223 & 15.270 \\
\hline$\delta_{\text {approx }}(\mathrm{mm})$ & 4.377 & 5.773 & 7.101 & 8.376 & 9.604 & 10.790 & 11.937 & 13.046 & 14.121 & 15.163 \\
\hline$\Delta(\%)$ & 1.889 & 2.895 & 2.924 & 2.667 & 2.336 & 2.006 & 1.713 & 1.431 & 1.167 & 0.930 \\
\hline
\end{tabular}

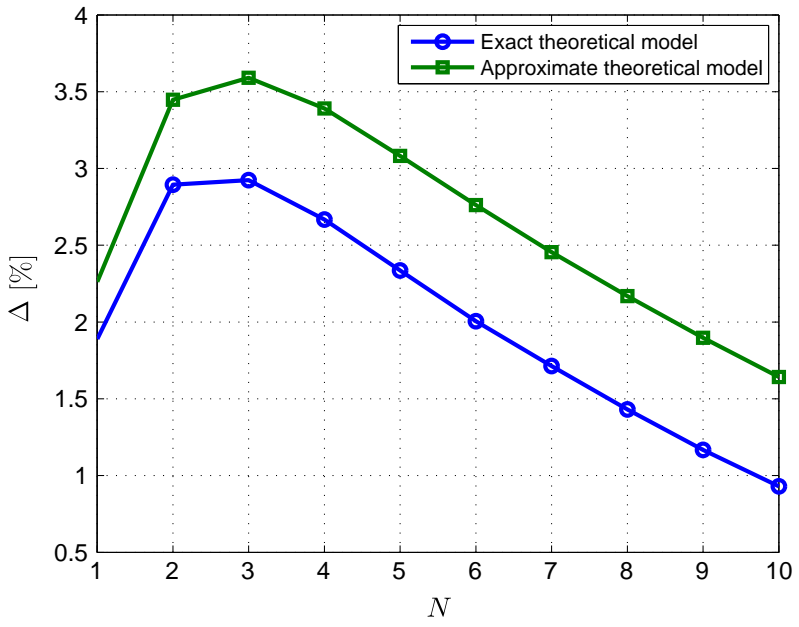

Fig. 9. Error $\Delta$ in terms of the exponent $N$, for the maximum deflection of a FGM beam in three-point bending.

(a) For the bending of cantilever beam by uniformly distributed force $q \quad 5 \mathrm{kN} \mathrm{m}^{1}$, we have employed 64 layers. The com parison of the results is presented in Table 1, for the values of the exponent $N=1,2, \ldots, 10$. We can observe a very good agreement between the analytical and the numerical results, since the errors range between $1 \%$ to $1 \%$. The percentage of relative error $\Delta$ is plotted in Fig. 6 , in terms of the exponent $N$.

(b) For the bending of the beam by a concentrated end force $P=5 \mathrm{kN}$, we have employed 128 layers. The concentrated force has been divided into equal parts acting in the nodes along the whole edge of the beam. This procedure reduces the concentration of stress in the numerical solution. The comparison between the analytical and the finite element solutions is shown in Table 2 . The errors $\Delta$ are very small: between $0.7 \%$ and $0.5 \%$. Fig. 7 presents the percentage of relative error, for the exact and approximate solutions, with respect to the numerical one.

From Figs. 6 and 7 we notice that the exact theoretical model gi ven by (79) is slightly better than the approximate one (in the least square sense). Moreover, we see that the approximate theoretical model (80) yields results in good agreement with the numerical and exact solutions, and it has the advantage of simplicity.

\subsection{Three point bending of functionally graded beam}

Let us consider the functionally graded beam described previ ously in relations (73) (80) subjected to three point bending. A concentrated central force $P=5 \mathrm{kN}$ acts at the mid span of the beam $\left(x_{3}=l / 2\right)$ in the $x_{1}$ direction, and the end edges $x_{3}=0, l$ are simply supported, see Fig. 8 . The analytical solution of this bending problem can be derived from the equations given in Section 3. For the maximum deflection $\delta$ of the beam, we get

$\delta_{\text {exact }} \frac{P l}{4}\left(\frac{1}{A_{1}}+\frac{l^{2}}{12 C_{2}}\right), \quad \delta_{\text {approx }} \quad \frac{P l}{4}\left(\frac{1}{\widetilde{A}_{1}}+\frac{l^{2}}{12 C_{2}}\right)$,

where the effective bending stiffness $C_{2}$ is given by (75), while the effective shear stiffness $A_{1}$ has the exact expression (79), and $\widetilde{A}_{1}$ is the approximate form (80).

To obtain the maximum deflection $\delta_{\mathrm{FEM}}$ by a finite element analysis, we use 128 layers to divide the beam domain. Table 3 shows the comparison of the theoretical and numerical solutions, together with the relative error $\Delta$. In Fig. 9 we plot the relative er ror with respect to the numerical solution, for $N=1, \ldots, 10$. We ob serve that the errors range between $0.9 \%$ and $2.9 \%$, depending on the value of $N$.

The shape of the beam in the deformed configuration is de picted in Fig. 10 for $N=1,5,10$, in both numerical and theoretical approaches. The results are in very good agreement, so that the curves for the analytical and numerical solutions are very close in Fig. 10. Indeed, according to Table 3, the relative errors for the

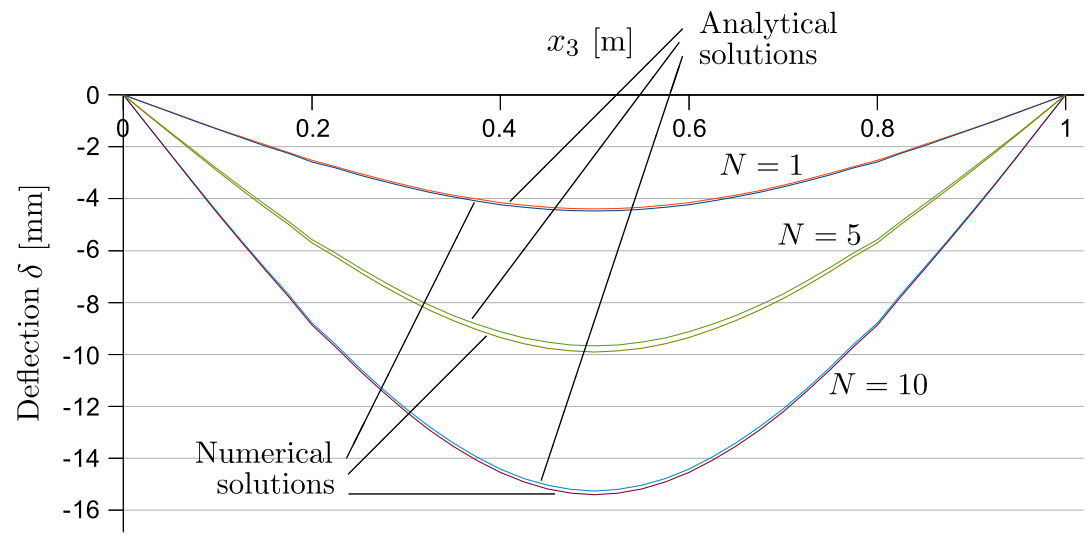

Fig. 10. Deflection of the FGM beam under three-point bending: numerical and analytical results, for the values of the exponent $N=1,5,10$. 


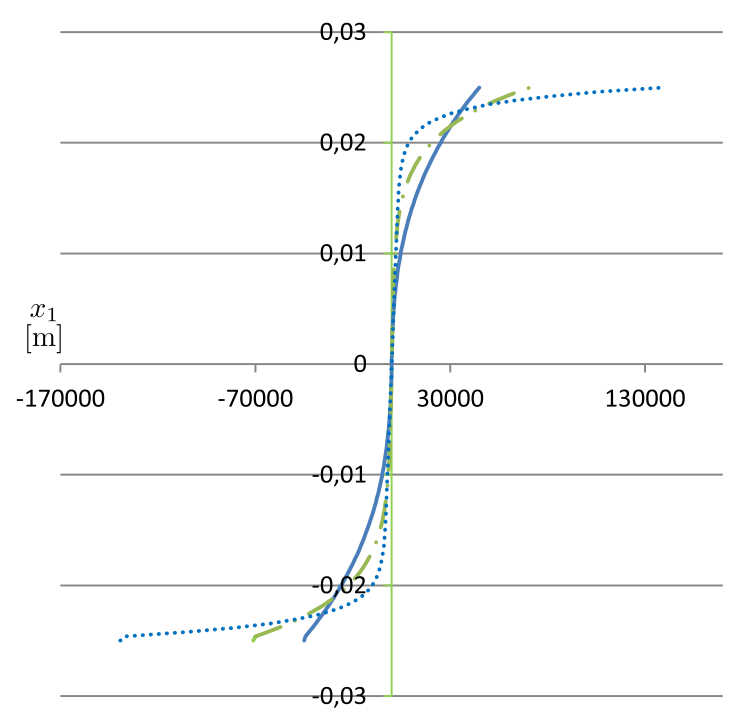

(a) Normal stress $[\mathrm{kPa}]$

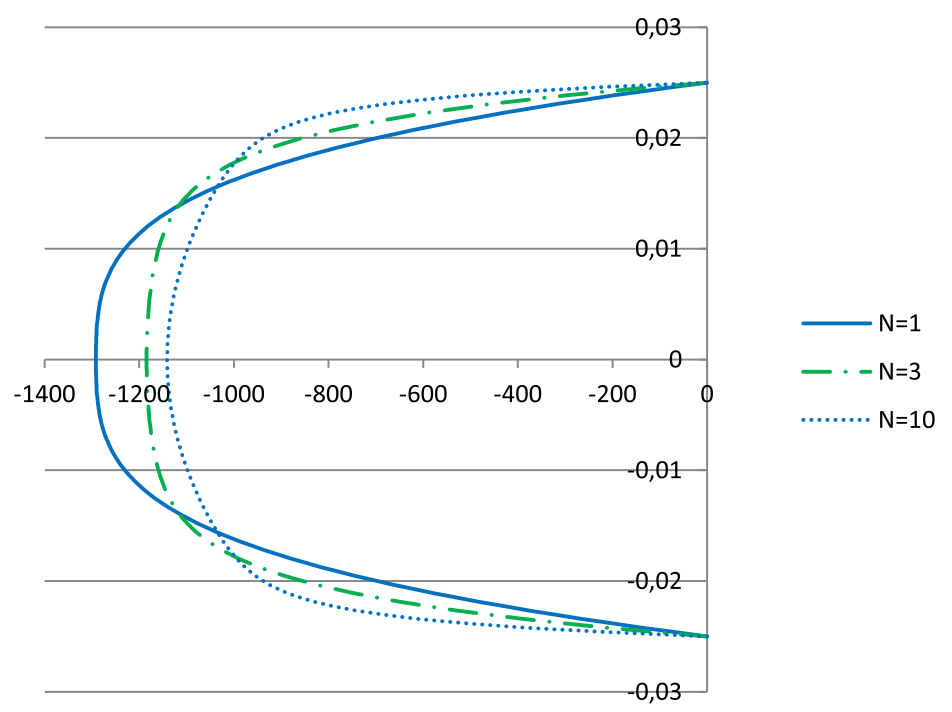

(b) Shear stress $[\mathrm{kPa}]$

Fig. 11. Distribution of normal and shear stresses in the cross-section of the FGM beam, for $x_{3}=l / 4$.

maximum deflections $\delta$ for $N=1,5,10$, are $1.8 \%, 2.3 \%$, and respec tively $0.9 \%$.

Let us present some results about the stress state in the FGM beam. For the cross section of the beam characterized by the axial coordinate $x_{3}=l / 4$, the distributions of the normal stress $t_{33}^{*}$ and shear stress $t_{31}^{*}$ versus the thickness coordinate $x_{1}$ are obtained by the finite element analysis and depicted in Fig. 11.

On the other hand, the analytical solution of this three point bending problem in the direct approach yields the following trans versal force $Q_{1}$ and bending moment $L_{1}$, calculated at the axial coordinate $x_{3}=l / 4$ :

$Q_{1} \frac{P}{2}, \quad L_{1} \quad \frac{P l}{8}$.

According to $(14)_{7,9}$, the correspondence between $Q_{1}, L_{1}$ and the three dimensional stress state is given by

$Q_{1} \quad b \int_{\frac{h}{2}}^{\frac{h}{2}} t_{31}^{*} \mathrm{~d} x_{1}, \quad L_{1} \quad b \int_{\frac{h}{2}}^{\frac{h}{2}} x_{1} t_{33}^{*} \mathrm{~d} x_{1}$.

Then, we can compare the theoretical predictions (83) with the numerical solution in the form of the resultants (84). As expected, the agreement between the two approaches is very good: for the bending moment $L_{1}$ the relative error is in the range 0.005 $0.007 \%$; for the transversal force $Q_{1}$ the relative error is about $0.00003 \%$ (for every exponent $N=1, \ldots, 10$ ).

\section{Conclusions}

In this paper we have employed the theory of directed curves to investigate the mechanical behavior of non homogeneous, com posite, and functionally graded beams. The structure of the consti tutive tensors and the form of the linear constitutive equations have been established in Sections 2, 3, and are presented in the relations (6) and (13). We determine the effective stiffness coeffi cients via comparison with three dimensional elasticity static and free vibration solutions in Sections 4 6. Thus, for non homo geneous isotropic beams we find the formulas (29) and (38), while for composite beams made of two different materials we have the effective stiffness properties (45). For orthotropic non homoge neous beams, the effective shear stiffness is expressed by (55),
(56), and the effective bending stiffness, extensional stiffness, tor sional rigidity and coupling coefficients are given by (50).

In Section 7 we apply these general formulas to determine the effective stiffness properties of some special functionally graded beams, such as orthotropic beams with exponential distribution law, or composite circular beams with power law distribution of material properties.

In Section 8 we consider rectangular functionally graded beams made of metal foams. Using the Gibson Ashby formula (74) for the Young modulus of closed cell aluminum foams, combined with the power law distribution of mass density (73), we find the effective stiffness coefficients in the form (75) and (79). In view of these re sults, we deduce the analytical beam like solutions for the bending of a FGM cantilever beam subjected to uniform and end loadings in Section 8.2, and for a FGM beam in three point bending in Section 8.3. The theoretical predictions are in good agreement with numer ical results obtained by a finite element analysis.

This comparison with finite element solutions represents a val idation of our analytical modeling concerning the effective stiffness properties of FGM beams. Nevertheless, our approach is much more general and it can be used to analyze the mechanical proper ties of various functionally graded rods, with different geometrical and material characteristics.

\section{Acknowledgments}

The authors acknowledge funding from the E.U. FP7 Programme FP7 REGPOT 20091 under Grant Agreement No. 245479, and from the Polish Ministry of Science and Higher Education, Grant No. 1471 1/7, PR UE/2010/7. In addition, the first author (M.B.) was supported by the Alexander von Humboldt Foundation, while the second author (H.A.) by the German Research Foundation (Grant AL341/33 1) and the Japanese Society for the Promotion of Science (ID No. RC 21115001). The fourth author (V.A.E.) acknowledges sup port from the German Research Foundation (Grant AL341/33 1).

\section{References}

[1] Trabucho L, Viaño JM. Mathematical modelling of rods. In: Ciarlet PG, Lions JL, editors. Handbook of numerical analysis, vol. 4. Amsterdam: North Holland; 1996. p. 487-974.

[2] Svetlitsky VA. Statics of rods. Berlin: Springer; 2000 
[3] Hodges DH. Nonlinear composite beam theory. Progress in astronautics and aeronautics, vol. 213. Reston: American Institute of Aeronautics and Astronautics Inc.; 2006.

[4] Meunier N. Recursive derivation of one-dimensional models from threedimensional nonlinear elasticity. Math Mech Solids 2008;13:172-94.

[5] Berdichevsky VL. Variational principles of continuum mechanics. II: applications. Heidelberg: Springer; 2009.

[6] Suresh S, Mortensen A. Functionally graded metals and metal-ceramic composites. 2: thermomechanical behaviour. Int Mater Rev 1997;42(3):85-116.

[7] Wang CM, Reddy JN, Lee KH. Shear deformable beams and shells. Amsterdam: Elsevier; 2000.

[8] Sankar BV. An elasticity solution for functionally graded beams. Compos Sci Technol 2001;61(5):689-96.

[9] Sankar BV, Tzeng JT. Thermal stresses in functionally graded beams. AIAA J 2002;40(6):1228-32.

[10] Chakraborty A, Gopalakrishnan S, Reddy JN. A new beam finite element for the analysis of functionally graded materials. Int J Mech Sci 2003;45(3):519-39.

[11] Chakraborty A, Gopalakrishnan S. A spectrally formulated finite element for wave propagation analysis in functionally graded beams. Int J Solids Struct 2003;40(10):2421-48.

[12] Giunta G, Belouettar S, Carrera E. Analysis of FGM beams by means of classical and advanced theories. Mech Adv Mater Struct 2010;17(8):622-35.

[13] Cosserat E, Cosserat F. Théorie des corps déformables. A. Herman et Fils, Paris; 1909.

[14] Ericksen JL, Truesdell C. Exact theory of stress and strain in rods and shells. Arch Ration Mech Anal 1958;1(1):295-323.

[15] Green AE, Naghdi PM. Non-isothermal theory of rods, plates and shells. Int J Solids Struct 1970;6:209-44.

[16] Green AE, Naghdi PM. On thermal effects in the theory of rods. Int J Solids Struct 1979;15:829-53.

[17] Antman SS. Nonlinear problems of elasticity. Series applied mathematical sciences, 107. New York: Springer; 1995.

[18] Rubin MB. Cosserat theories: shells, rods, and points. Dordrecht: Kluwer Academic Publishers.; 2000.

[19] Zhilin PA. Mechanics of deformable directed surfaces. Int J Solids Struct 1976;12:635-48.

[20] Zhilin PA. Nonlinear theory of thin rods. In: Indeitsev DA, Ivanova EA, Krivtsov AM, editors. Advanced problems in mechanics, vol. 2. St. Petersburg: Problems Mech. Eng. R.A.S. Publ.; 2006. p. 227-49.

[21] Zhilin PA. Applied mechanics - theory of thin elastic rods (in Russian). Petersburg: Politekhn. Univ. Publ., St.; 2007.

[22] Altenbach H, Naumenko K, Zhilin PA. A direct approach to the formulation of constitutive equations for rods and shells. In: Pietraszkiewicz W, Szymczak C, editors. Shell structures: theory and applications. London: Taylor and Francis; 2006. p. 87-90.

[23] Ieşan D. Classical and generalized models of elastic rods. Boca Raton - London New York: Chapman \& Hall/CRC Press; 2009. cylinders. J Elast 2007;87:147-59.

[25] Gibson LJ, Ashby MF. Cellular solids: structure and properties. Cambridge solid state science series. Cambridge: Cambridge University Press; 1997.

[26] Lurie AI. Theory of elasticity. Berlin: Springer; 2005.

[27] Lebedev LP, Cloud MJ, Eremeyev VA. Tensor analysis with applications in mechanics. New Jersey: World Scientific; 2010.

[28] Zhilin PA. Applied mechanics - foundations of shell theory (in Russian). Petersburg: Politekhn. Univ. Publ., St.; 2006.

[29] Zelenina AA, Zubov LM. The non-linear theory of the pure bending of prismatic elastic solids. J Appl Math Mech 2000;64(3):399-406.

[30] Zubov LM. Exact nonlinear theory of tension and torsion of helical springs. Doklady Phys 2002;47(8):623-6.

[31] Yu W, Hodges DH, Volovoi V, Cesnik CES. On Timoshenko-like modeling of initially curved and twisted composite beams. Int J Solids Struct 2002;39(19):5101-21.

[32] Marigo J-J, Meunier N. Hierarchy of one-dimensional models in nonlinear elasticity. J Elast 2006;83(1):1-28.

[33] Mora MG, Müller S. Convergence of equilibria of three-dimensional thin elastic beams. Proc Royal Soc Edinburgh: Sect A Math 2008;138:873-96.

[34] Scardia L. Asymptotic models for curved rods derived from nonlinear elasticity by $\Gamma$-convergence. Proc Royal Soc Edinburgh: Sect A Math 2009;139:1037-70.

[35] Irschik H, Gerstmayr J. A continuum mechanics based derivation of Reissner's large-displacement finite-strain beam theory: the case of plane deformations of originally straight Bernoulli-Euler beams. Acta Mech 2009;206:1-21.

[36] Humer A, Irschik H. Onset of transient vibrations of axially moving beams with large displacements, finite deformations and an initially unknown length of the reference configuration. ZAMM 2009;89(4):267-78.

[37] Irschik H, Gerstmayr J. A continuum-mechanics interpretation of Reissner's non-linear shear-deformable beam theory. Math Comput Model Dynam Syst 2011;17(1):19-29.

[38] Muskhelishvili NI. Some basic problems of the mathematical theory of elasticity. Groningen: Noordhoff; 1953.

[39] Solomon L. Élasticité Linéaire. Paris: Masson; 1968.

[40] Timoshenko SP. On the correction for shear of the differential equation for transverse vibrations of prismatic beams. Phil Mag 1921;41:744-6.

[41] Lomakin VA. Theory of nonhomogeneous elastic bodies (in Russian). MGU, Moscow; 1976.

[42] Lekhnitskii SG. Elementary solutions of two special problems of equilibrium of nonhomogeneous cylinders (in Russian). Investigations on elasticity and plasticity, vol. 6. Petersburg: Izd. Leningrad Univ., St.; 1967.

[43] Altenbach H, Öchsner A. Cellular and porous materials in structures and processes. CISM courses and lectures, vol. 521. Wien NewYork: Springer; 2010.

[44] Altenbach H, Eremeyev VA. Direct approach-based analysis of plates composed of functionally graded materials. Arch Appl Mech 2008;78:775-94. 